\title{
Atmospheric Variability in a Coupled Atmosphere-Ocean-Sea Ice Model with Interactive Stratospheric Ozone Chemistry
}

\author{
S. Brand", K. Dethloff and D. Handorf \\ Alfred Wegener Institute for Polar and Marine Research, Research Unit Potsdam, Potsdam, Germany
}

\begin{abstract}
Stratospheric ozone is an important factor impacting on climate dynamics and thus on atmospheric variability. Aiming to develop a better general understanding of chemistry-dynamics feedbacks, we applied a coupled atmosphereocean-sea ice general circulation model (AOGCM) with interactive chemistry, extending up to $80 \mathrm{~km}$. With this model, ECHO-GiSP, two 150-year climate simulations were performed in order to enable us to focus on coupling mechanisms between stratospheric ozone chemistry and dynamical processes on interannual to decadal scales. Our results from these simulations indicate significant circulation changes in the tropo- and stratosphere due to interactive stratospheric ozone feedbacks, which were enabled in the 'interactive run' compared to the 'reference run'. We discuss the decadal variability within the runs by analysing the Arctic Oscillation (AO) mode. In particular, the tropospheric variability within the interactive simulation, which tends to the negative AO phase, appears to be enhanced, while the stratospheric/mesospheric variability weakens.
\end{abstract}

\section{INTRODUCTION}

The global atmospheric circulation is effected by incoming solar radiation, and thus differential warming of the atmosphere. Besides the earth's surface at the lower boundary mainly middle atmosphere greenhouse gases, especially ozone, are controlling this radiative heating by absorption of solar ultraviolet (UV) and terrestrial infrared (IR) radiation. The resulting stratospheric temperature distribution and dynamical structures interact with the tropospheric ones, leading to a complex radiation-dynamics equilibrium. Thus changes in greenhouse gas concentrations and distributions, be that due to natural variability or anthropogenic influence, are likely to exert a strong impact on global climate. Since, in turn, stratospheric ozone formation and destruction are highly temperature dependent, also dynamical changes are significantly affecting atmospheric chemistry. For example, a cooling in the stratosphere, as connected with a global tropospheric warming, would slow down the overall stratospheric gas phase chemistry, but at the same time enhance catalytic ozone destruction in the polar lower stratosphere, where heterogenous chlorine activation on Polar Stratospheric Clouds (PSC's) would be intensified. To account for such complex, nonlinear dependencies a coupled atmosphere-ocean-sea ice model including interactive chemistry is needed.

Earlier climate studies focused on middle atmosphere dynamics were applying atmospheric general circulation models (GCM's) with different parameterizations of most important prescribed chemistry feedbacks (e.g. Manzini and Bengtsson, Shindell et al. [1-3]). First integrations including interactive chemistry have been carried out by Eckman et al. [4] for short time slices. Later, similar simulations were done by Austin et al. and Austin [5, 6], while Dameris et al. [7] and Hein et al. [8] focused on the lower stratosphere due to a

*Address correspondence to this author at the Alfred Wegener Institute for Polar and Marine Research, Research Unit Potsdam, Potsdam, Germany;

E-mail: sbrand@awi-potsdam.de model top of about $30 \mathrm{~km}$. Although basically this was sufficient to reproduce most of the observed variability and characteristics, a model top in the middle stratosphere implies the problem of 'cutting' important upper stratospheric parts of the Brewer-Dobson meridional mean circulation (BDC), and thus preventing downward mass transport in polar latitudes. This 'downward control' mechanism affecting temperatures and greenhouse gas concentrations in the polar vortex (see Holton et al., Haynes et al., Rind et al. [9-11]) should therefore be included in middle atmosphere modeling by using higher model tops. A first instructive study carried out with a model extending up to $80 \mathrm{~km}$ and taking into account several 20-year runs, though focussing specially on atmospheric chemistry features, was presented by Steil et al. [12].

Here we present results from two 150-year long simulations with the coupled AOGCM ECHO-GiSP with a model top at $80 \mathrm{~km}$ height including the middle atmosphere and interactive stratospheric chemistry. For the first time such simulations of this duration are carried out using a fully coupled atmosphere-ocean-sea ice model. Since thereby the atmospheric boundary conditions are interactively provided by the linked ocean-sea ice model, important dynamical coupling processes as adressed by Latif et al., von Storch, Dethloff et al. [13-15] or Rinke et al. [16] are taken into consideration. A reasonable coupling between the atmosphereand ocean-sea ice component appears to be the necessary prerequisite in order to study coupling mechanisms and decadal variability including also interactive feedbacks between chemistry and dynamics. The model as used for this study is able to produce decadal scale variability and to capture most of the observed stratospheric structures (Huebener et al. [17]). The applied chemistry scheme can easily be adjusted to other research questions for future simulations.

Following an overview regarding the model and simulation set up (section 2), selected results are presented in section 3. Section 4 then provides a discussion with respect to some aspects of these results, as well as general conclusions are drawn. 


\section{MODEL AND EXPERIMENTS}

ECHO-GiSP, which is the abbreviation for 'ECHO-G with Integrated Stratospheric chemistry by AWI Research Unit Potsdam', is an extension of the coupled atmosphereocean general circulation model ECHO-G ('ECHAM \& HOPE-G', Legutke and Voss [18]). The chemistry part of this newly developed model is based on the MECCA chemistry module ('Module Efficiently Calculating the Chemistry of the Atmosphere', see Sander et al. [19]). Since ozone chemistry plays especially in the stratosphere a major role regarding the impact of atmospheric chemistry on radiation and thus also on dynamics, this implies to use a model version, that has to cover not only the troposphere, but also the stratosphere. Therefore the 39-level middle atmosphere version of the ECHO-G with its upper level at $1 \mathrm{~Pa}(80 \mathrm{~km})$ was used in ECHO-GiSP. Additionally, as stated before, a low model top could lead to problems with the 'downward control' mechanism at polar latitudes.

ECHO-G, one main part of ECHO-GiSP, includes the atmosphere model ECHAM4 and the ocean model HOPE-G as components, which are coupled via the OASIS coupler ('Ocean Atmosphere Sea Ice Soil coupler', Valcke et al. [20]). ECHAM4, described by Roeckner et al. [21], is a climate model version of the ECMWF model, which was tuned and extended to the middle atmosphere ('MA'-ECHAM4, for details see Manzini et al. [22] and Manzini and McFarlane [23]). It is a spectral model, including state-of-the-art parameterizations for different physical processes, like e.g. radiative transfer, cumulus convection, gravity wave drag, boundary layer and free atmosphere turbulence. HOPE-G ('Hamburg Ocean Primitive Equation model on Global scale', Wolff et al., Legutke and Maier-Reimer [24, 25]) is a primitive equation model with 20 vertical levels, that contains a dynamic-thermodynamic sea ice model with snow cover. Coupling is enabled by exchanging sea surface temperatures (SST's) and surface fluxes between the two standalone GCM's, including a flux correction applied for heat and freshwater exchange. The 'coupled' time step for this exchange is a multiplier of the atmosphere-, as well as the ocean time step. The time steps used for this study are 15 minutes for the atmosphere, 2 hours for the ocean and 1 day for the coupling, where the horizontal resolutions are T30 (triangular truncation at wavenumber $30, \sim 3.75^{\circ} \times 3.75^{\circ}$ ) for the atmosphere, respective T42 for the ocean $\left(2.8^{\circ} \times 2.8^{\circ}\right)$, with a refinement towards the equator. The 39 atmospheric levels from the surface to $0.01 \mathrm{hPa}(80 \mathrm{~km})$ have a vertical grid distance of about $2 \mathrm{~km}$ in the lower stratosphere, while the resolution gets coarser towards the top.

MECCA, the chemistry module of ECHO-GiSP, calculates atmospheric chemistry at each gridpoint of a given model grid (see Sander et al. [19]). It is switched to ECHOGiSP by a newly developed coupling routine. In the standard MECCA environment a range of predefined chemistry configurations with choices of chemical species and reactions are available and can be integrated into the source code via the KPP program ('Kinetic PreProcessor', Damian-Iordache and Sandu [26], Damian et al. [27]). The new environment additionally supports a free choice of the configuration of chemical species and reactions, where only the desired setup has to be specified.
Technically, the mixing ratios for the defined chemical species and some of the standard meteorological variables (temperature, pressure etc.), that influence the ongoing chemical reactions, are exchanged between ECHO-G and MECCA every atmospheric time step. The module calculates the reaction rate constants for photolysis reactions (following Landgraf and Crutzen [28]) and for reactions on Polar Stratospheric Clouds (PSC's), and uses these reaction rate constants, as well as the reaction rate constants for the 'ordinary' gas phase reactions, to predict the chemistry mixing ratios for the next time step. Back within ECHO-G, the mixing ratios for all species are transported via the semiLagrangian transport scheme by Rasch and Williamson [29]. In such a way the (non-interactive) coupling from the model dynamics to chemical model changes is realized. Additionally, the interactive two way feedback between both components can be enabled by using the MECCA-trace gas concentrations within the ECHO-G radiation scheme (Morcrette [30]), instead of the predefined climatological 'ECHAM'trace gas concentrations.

The chemistry configuration for the simulations within this study is a setup with 39 chemical species, for which 116 chemical reactions are defined (81 gas phase reactions, 25 photolysis reactions and 10 heterogenous reactions on PSC's). The species include the main members of the OX, NOX, ClOX, HOX and BrOX chemical families, as well as other atmospheric gases like $\mathrm{CO}, \mathrm{CO} 2, \mathrm{CH} 4, \mathrm{~N} 2, \mathrm{H} 2$. $\mathrm{H} 2 \mathrm{O}$ is also present in the chemistry scheme, but can even in the interactive model version not feed back to the radiation. This means, that the $\mathrm{H} 2 \mathrm{O}$ mixing ratio from $\mathrm{ECHO}-\mathrm{G}$ is taken into account within MECCA, but is not recalculated and given back to the atmosphere model.

Since our main focus is on stratospheric chemistry, data from the KASIMA chemistry transport model ('KArlsruhe SImulation model of the Middle Atmosphere', Kouker et al. [31]) have been used to provide the boundary conditions in the troposphere, as well as the initial conditions on the entire grid. The use of this model setup allows interactive chemistry and dynamical feedbacks in the stratosphere, while keeping prescribed chemistry in the troposphere in order to avoid a too complex chemistry scheme.

For this study we used ECHO-GiSP to perform two 150year simulations: An equilibrium run in the non-interactive mode, further called 'reference run', and a simulation under the same initial and boundary conditions, but in the interactive chemical-dynamical mode, refered to as 'coupled run'. The reference run applied the stratospheric chemistry only in a passive way, driven by the simulated dynamics but with prescribed conditions for the radiation. The coupled run additionally considered interactive chemistry-dynamics feedbacks by replacing the fixed climatological ozone concentrations in the radiation scheme with the modeled ones. Thus, in the coupled simulation the radiative heating component due to the absorption of stratospheric ozone is fully time dependent and controlled only by the model dynamics.

\section{RESULTS}

Prior to the discussion of the differences between the two simulations performed for this study it should also be noted, that they show convincing agreements in general features. All model variables indicate a high level of dynamic coinci- 
dences between both simulations and in particular a reasonable behaviour compared to measurements (see e.g. Huebener et al. [17]). The validation shows, that the tropospheric and stratospheric jets occur with the right position and strenght in the model, which also forms a well developed polar vortex. Further, the tropospheric circulation cells and the interhemispheric Brewer-Dobson meridional mean circulation in stratosphere and mesosphere appear realistic. The temperatures show a warm troposphere in general and a cold lower stratosphere especially in the tropics, the model mesopause is located at around $1 \mathrm{hPa}(45$ to $50 \mathrm{~km}$ ). Moreover, there is a latitude dependent tropopause height of the right size and reasonable large scale ascent and descent. A detailed discussion especially of a few weak points in the atmospheric model part can also be found in Manzini and McFarlane [23].

In the following we present our results divided into a comparison between coupled and reference simulation in section 3.1, and an evaluation of internal variability within the simulations in section 3.2, focusing on the Arctic Oscillation (AO), one of the most important teleconnection patterns, introduced by Thompson and Wallace [32].

\subsection{Comparison between the Coupled- and the Reference Simulation}

In this study we focus on the northern hemisphere winter period (DJF) as the main important one with respect to a reasonable signal-to-noise ratio of possible changes. We chose a spin-up time of 30 years. Therefore only the model years 31 to 150 were used to calculate 120 -year means for each of the runs, in order to avoid the representation of transition effects, as they could appear while approaching an equilibrated mean state from the given starting conditions for the different model parts (atmosphere, chemistry, ocean, sea ice). Principally, 10 to 15 years of equilibration would be enough regarding the atmosphere, but coupling with an interactive ocean component requires a longer adjustment phase, although we used an already equilibrated initial state from an earlier ECHO-G control run.

Note, that all the means were calculated via monthly values. This additional step allowed to extract the associated standard deviations, representing timescales starting from seasonal. The standard deviations were used to perform Student's t-tests for the differences of the means between the simulations. Results of this tests are given as $95 \%$ significance levels in the figures, contoured by white lines.

The Fig. (1a,b) show the mean sea level pressure (mslp) of the northern hemisphere (NH) for the coupled and the reference run. The patterns are in a rather good agreement, with a tendency to a stronger Aleutian low in the coupled run. This is a hint on a change of the AO mode (Thompson and Wallace [32]) towards the negative phase. Area means of the mslp have been computed for both simulations, the deviations from these means are shown in the Fig. $(\mathbf{2 a}, \mathbf{b})$, suggesting that the runs are similar stable in general. Further, in order to avoid the limitations in time-frequency localization, which would occur 'simply' performing Fourier power spectra, the timeseries were analysed using wavelet transform spectra (Torrence and Compo [33]). This spectra for the coupled and the reference run (Fig. 3a,b) show similar

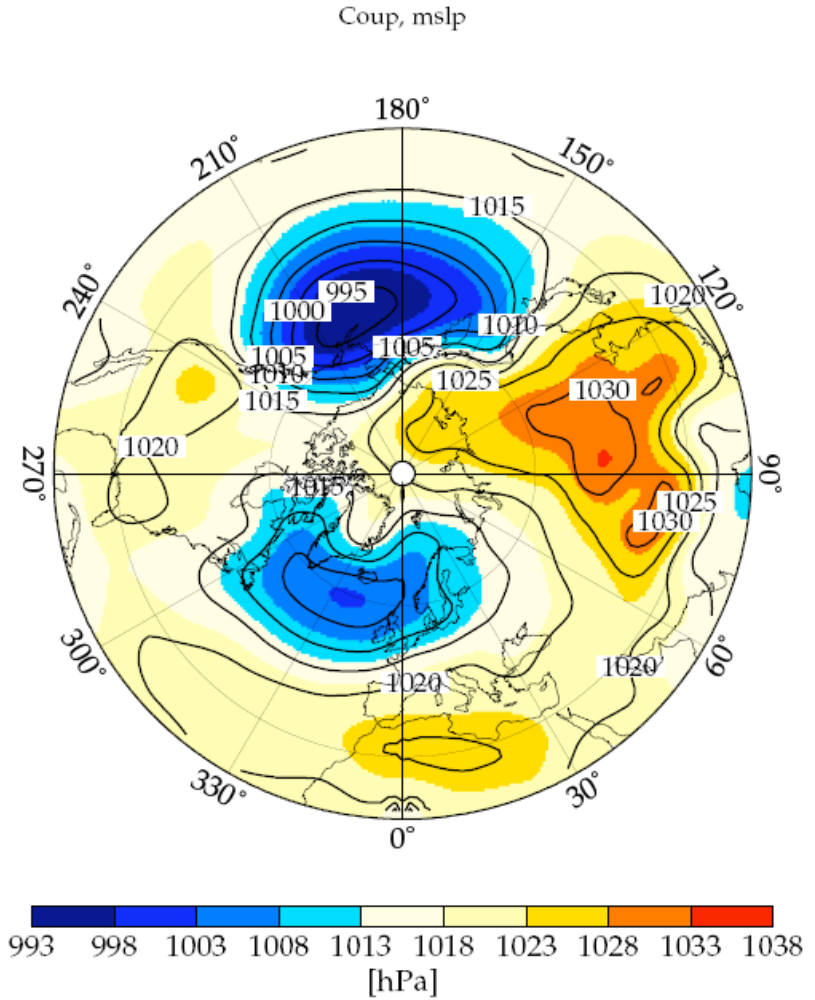

Fig. (1a). Mean sea level pressure (mslp) in [hPa] for DJF. Shown is the average over the 120 model years 31 to 150 for the coupled run.

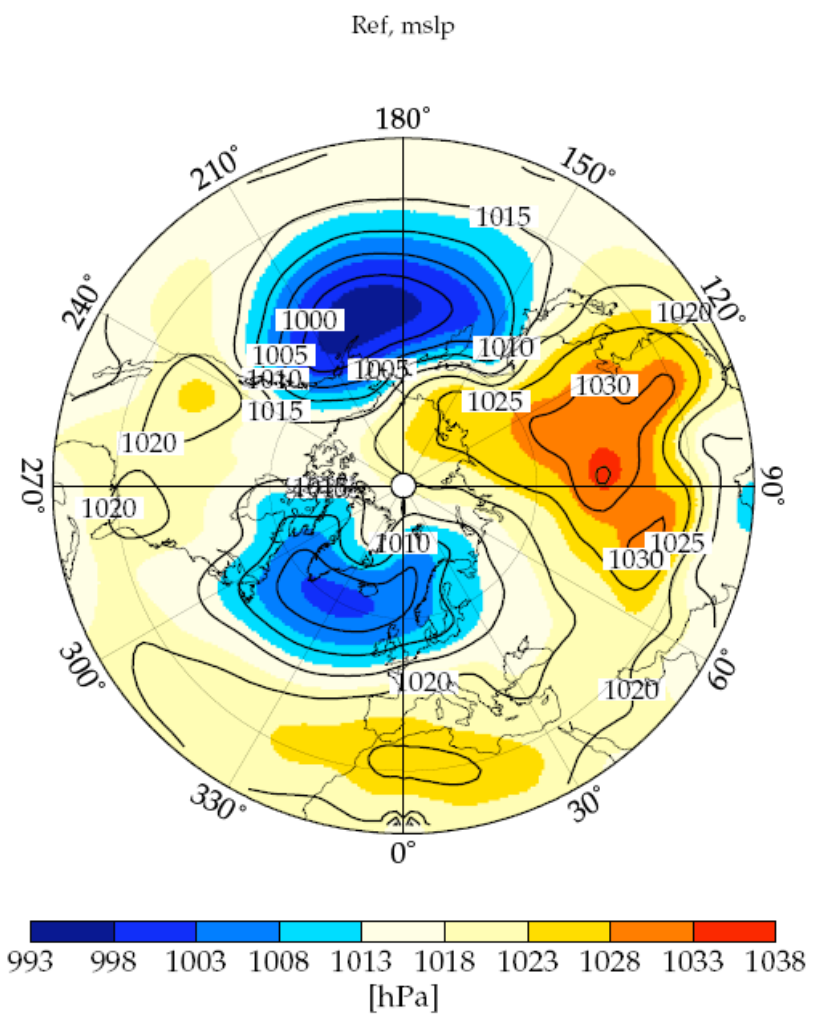

Fig. (1b). Mean sea level pressure (mslp) in $[\mathrm{hPa}]$ for DJF. Shown is the average over the 120 model years 31 to 150 for the reference run. 


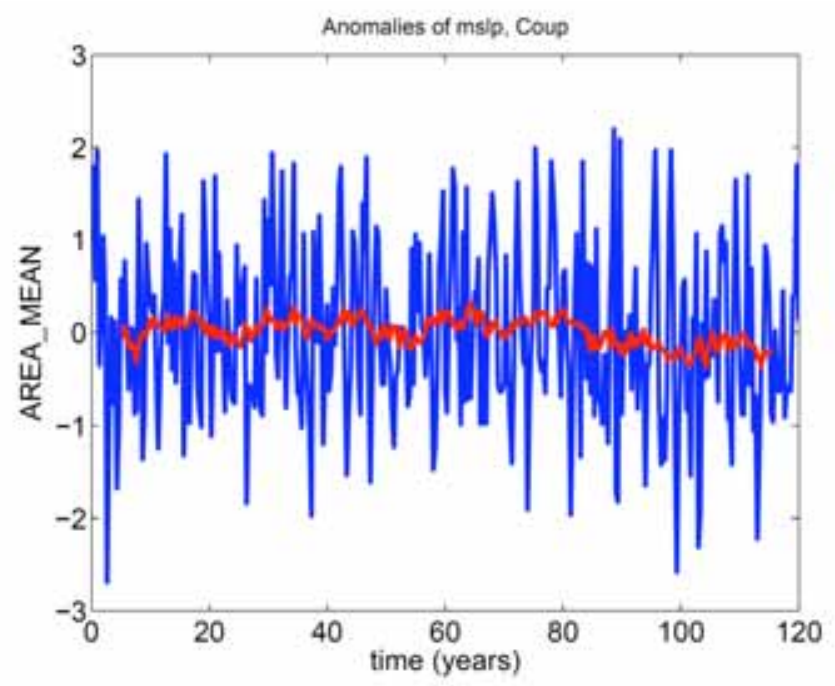

Fig. (2a). Mean sea level pressure (mslp) for DJF. Time evolution of the deviations (in $[\mathrm{hPa}]$ ) from the area means for the model years 31 to 150 . Coupled run.

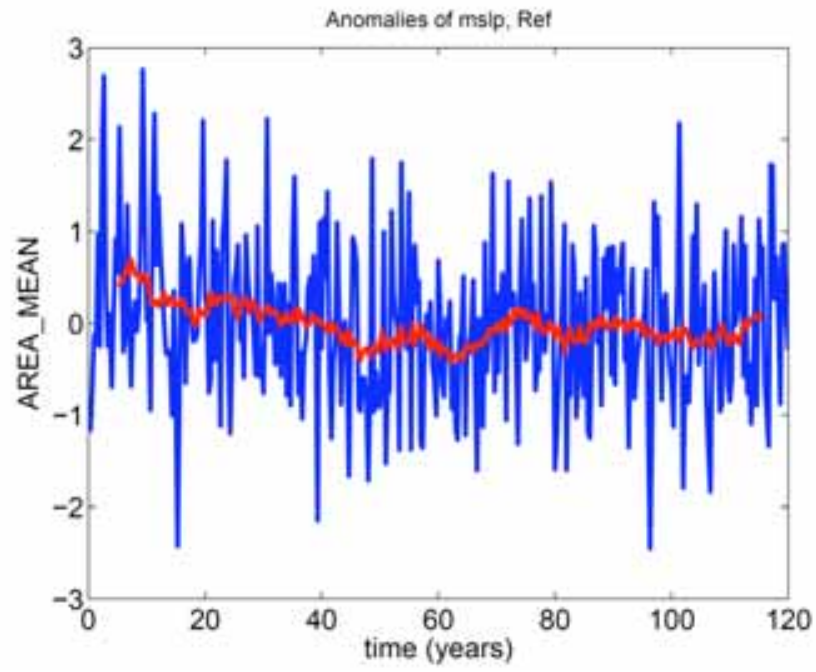

Fig. (2b). Mean sea level pressure (mslp) for DJF. Time evolution of the deviations (in $[\mathrm{hPa}]$ ) from the area means for the model years 31 to 150 . Reference run.

variability for both simulations, although on interannual time scales there seems to be a tendency to slightly increased variability in the coupled run.

In the Fig. (4a-d) the differences between the coupled and the reference simulation for the mslp, and for the geopotential heights at $200 \mathrm{hPa}, 10 \mathrm{hPa}$ and $1 \mathrm{hPa}$ are presented. The tropospheric differences show an inverse AO-like pattern with negative northern pacific, positive polar and negative northern atlantic deviations. Thus the coupled simulation favours a more negative AO phase with a weaker Icelandic low and Azoric high, which is also related to a more negative phase of the North Atlantic Oscillation (NAO, Hurrell [34]). The latter is connected with a warmer, more disturbed stratospheric vortex for the coupled run compared to a colder and stronger polar vortex for the reference run. Within the troposphere it is of interest to recognize the anticorrelation between the strength of the climatological pressure systems in the atlantic and in the pacific sector due to the AO signal. Another interesting feature, which is characteristic for the
$\mathrm{AO}$, is the almost barotropic appearence of the described differences within the troposphere. In the upper stratosphere the differences between the simulations form a dipole structure, which indicates an increase of the planetary wavenumber 1 in the coupled run.

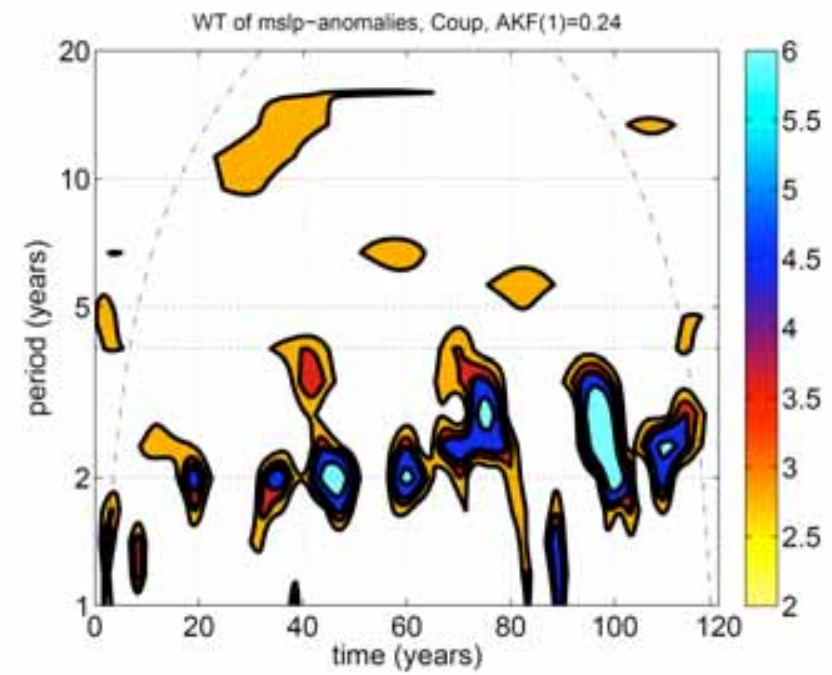

Fig. (3a). Mean sea level pressure (mslp) for DJF. Local wavelet power spectra of the time series from Fig. (2a), obtained with the Morlet wavelet. At both ends, dash-dotted lines separate regions where edge effects become important. The thick black contour envelopes areas exceeding the $95 \%$ confidence level for a corresponding red noise process. Coupled run.

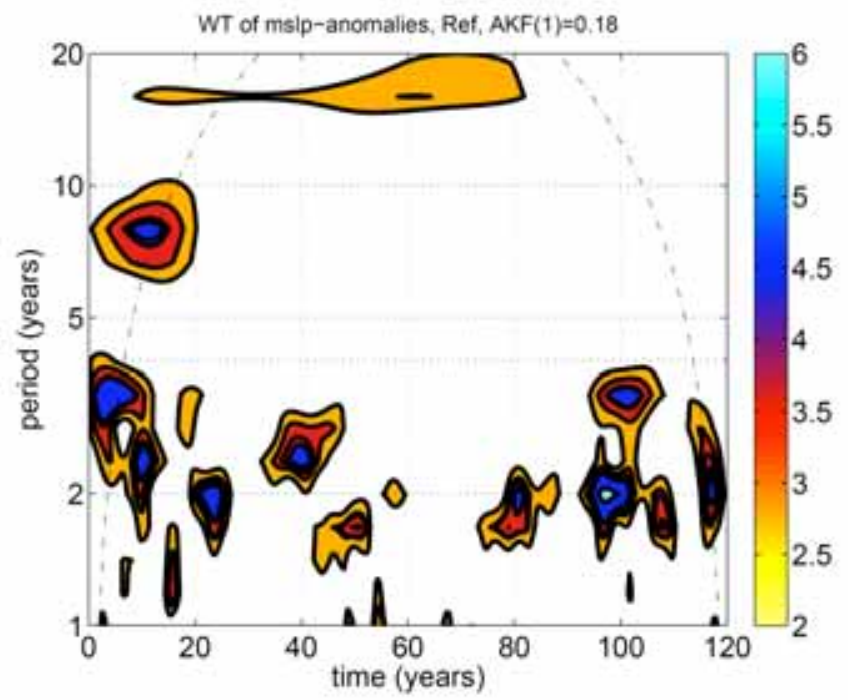

Fig. (3b). Mean sea level pressure (mslp) for DJF. Local wavelet power spectra of the time series from Fig. (2b), obtained with the Morlet wavelet. At both ends, dash-dotted lines separate regions where edge effects become important. The thick black contour envelopes areas exceeding the $95 \%$ confidence level for a corresponding red noise process. Reference run.

The zonal mean winds for the coupled and the reference simulation (Fig. 5a,b) indicate clear systematic changes in the stratosphere, as well as in the troposphere. There is a weaker polar stratospheric jet in the coupled run, while the easterly stratospheric jet occurs more similar for both runs, showing extreme values up to $65 \mathrm{~m} / \mathrm{s}$. In the troposphere the winter subtropical jet, located at about $35^{\circ} \mathrm{N}$, has mean 


\section{MEAN Coup - Ref}
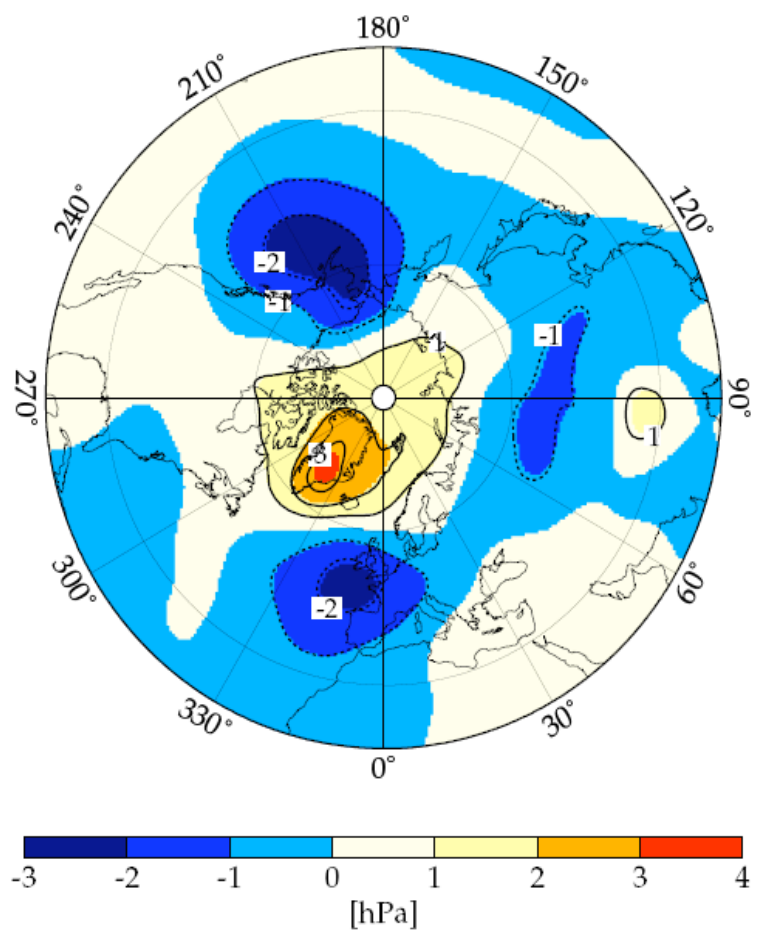

Fig. (4a). Differences between coupled- and reference run (in [hPa]) for the mean sea level pressure, averaged for DJF of the model years 31 to 150 .

MEAN Coup - Ref
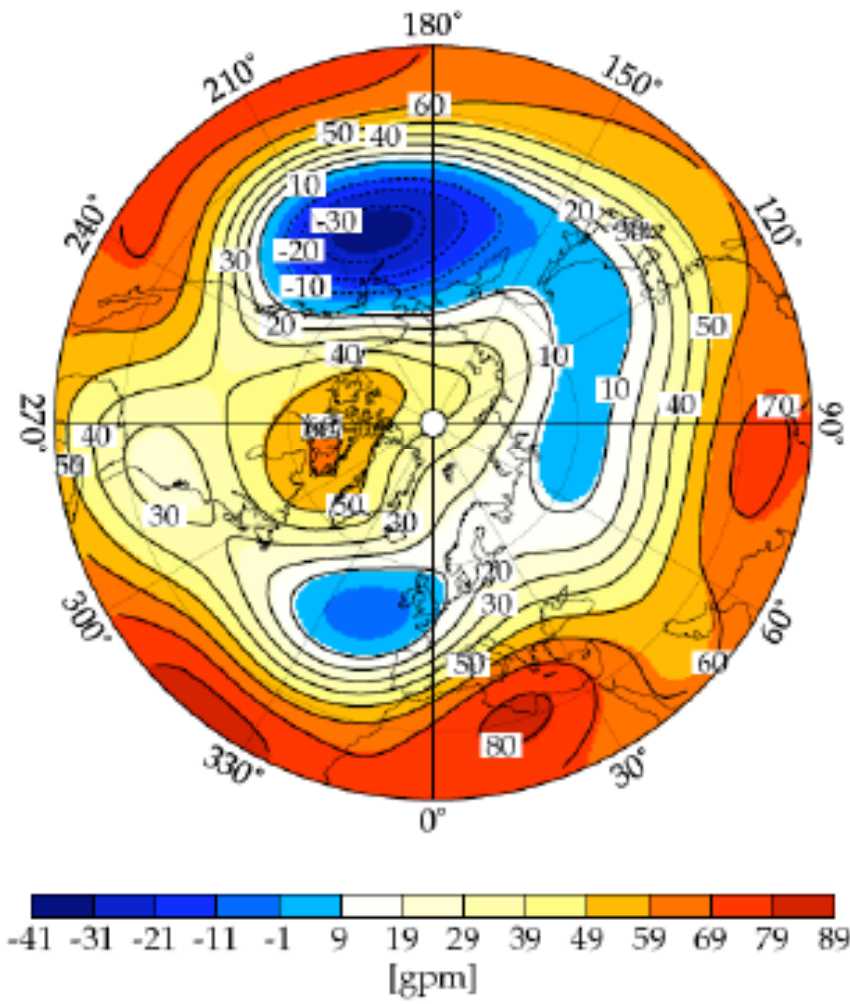

Fig. (4b). Differences between coupled- and reference run (in [gpm]) for the geopotential height field at $200 \mathrm{hPa}$, averaged for DJF of the model years 31 to 150 .
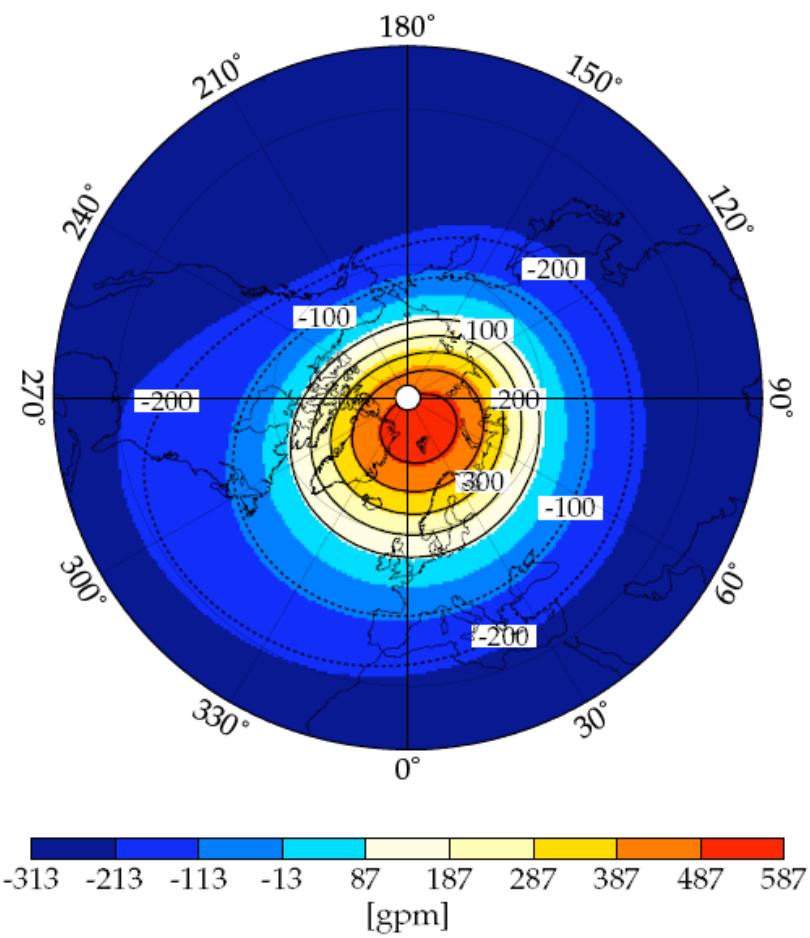

Fig. (4c). Differences between coupled- and reference run (in [gpm]) for the geopotential height field at $10 \mathrm{hPa}$, averaged for DJF of the model years 31 to 150 .

MEAN Coup - Ref
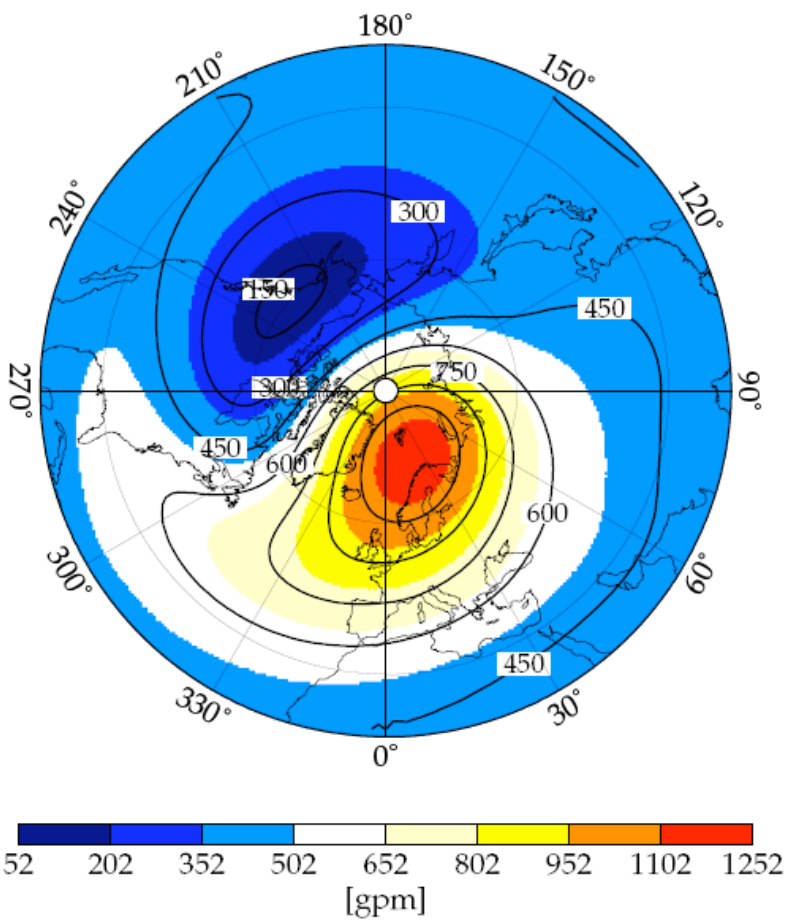

Fig. (4d). Differences between coupled- and reference run (in [gpm]) for the geopotential height field at $1 \mathrm{hPa}$, averaged for DJF of the model years 31 to 150 . 
maximum values of more than 40 to $45 \mathrm{~m} / \mathrm{s}$ in both simulations. The summer jet at $40^{\circ} \mathrm{S}$ stays below $40 \mathrm{~m} / \mathrm{s}$. Nevertheless there is a strenghtening of about 10 to $15 \%$ in the coupled simulation compared to the reference run. The associated weakening of the polar stratospheric jet amounts up to $30 \%$. For the easterly stratospheric jet in the summer hemisphere a slight 'reshaping' of the pattern can be determined (Fig. 5c): While the mean maximum of the windspeed is stretched more vertically for the reference run, it distributes more horizontally for the coupled run. This 'reshaping' effect has high corresponding significances and indicates a stabilizing of the vertical stratospheric stratification in the coupled run.

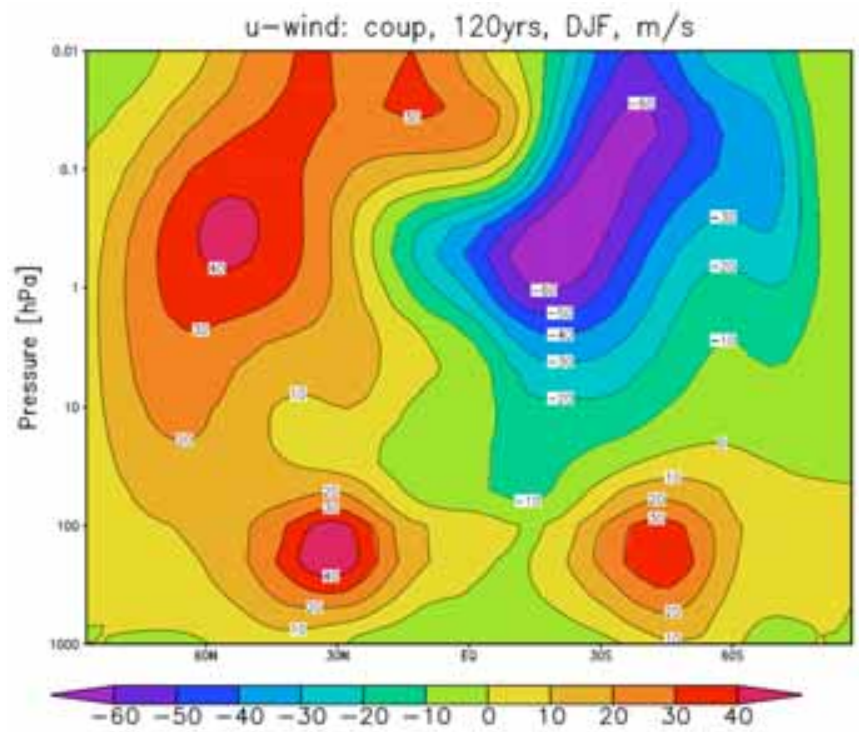

Fig. (5a). Zonal mean of the zonal wind $u$ in $[\mathrm{m} / \mathrm{s}]$, averaged for DJF of the model years 31 to 150 . Coupled run.

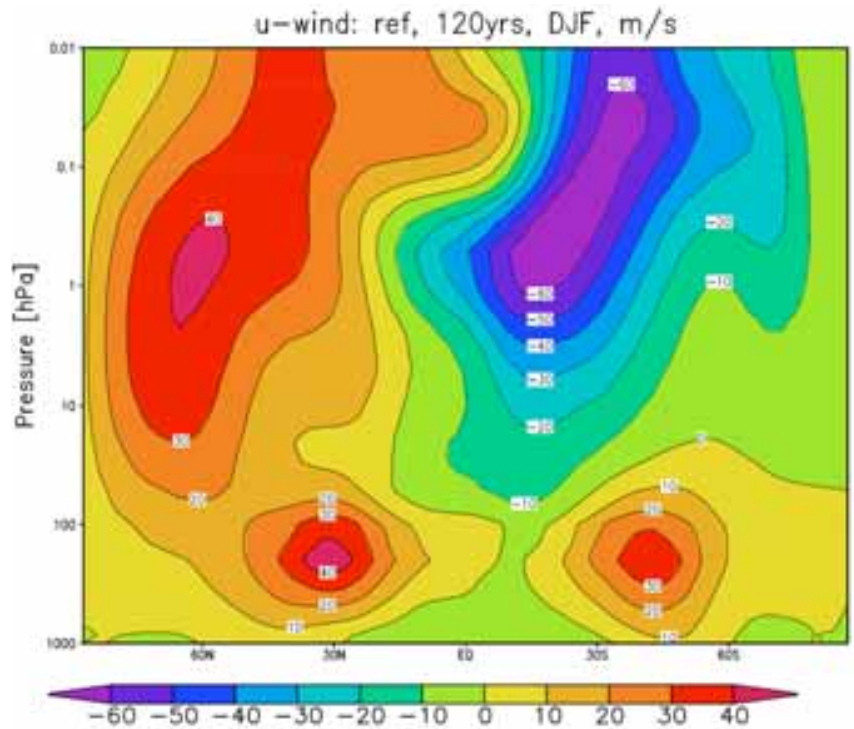

Fig. (5b). Zonal mean of the zonal wind $u$ in $[\mathrm{m} / \mathrm{s}]$, averaged for DJF of the model years 31 to 150 . Reference run.

Proceeding to the ozone concentrations shown in the Fig. $(\mathbf{6 a}, \mathbf{b})$ for the coupled run and the reference run, it first has to be pointed out again, that the changes between the simulations are only due to the fact, that in the coupled run the model is free to go into its real physical-chemical equilib-

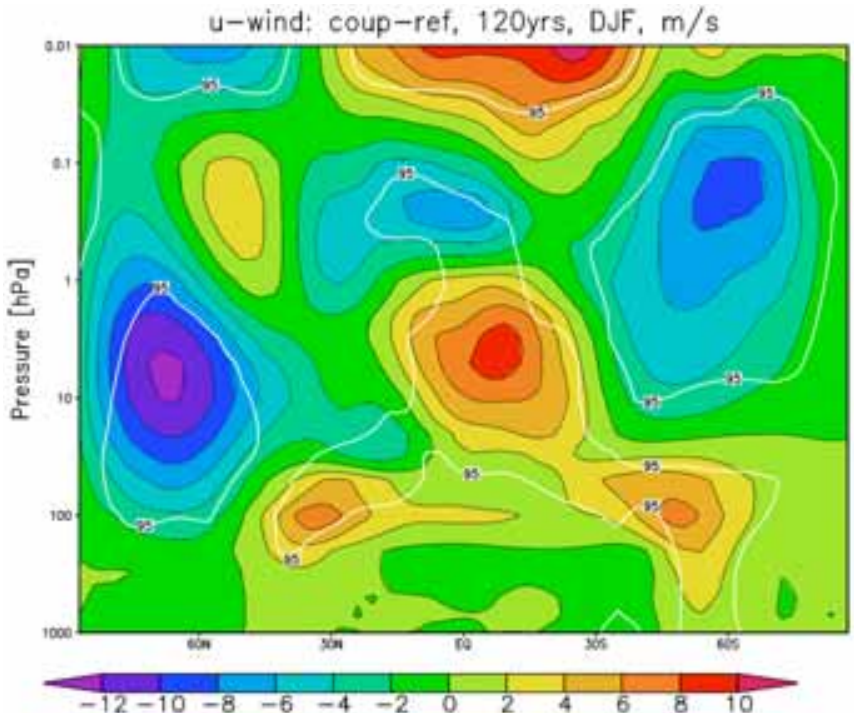

Fig. $(\mathbf{5 c})$. Zonal mean of the zonal wind $\mathrm{u}$ in $[\mathrm{m} / \mathrm{s}]$, averaged for DJF of the model years 31 to 150 . Differences between coupledand reference run.

rium state. In the reference run this is not the case, since there the model is forced by the (principally arbitrary) choice of the ozone profiles used within the model radiation scheme. For this study it is of central importance just to note, that there are clear changes because of the consideration of interactive stratospheric chemistry, and that these are the basic reason of all the dynamical changes between the simulations.

The maximum ozone values appear slightly below 9 ppmv in the coupled simulation (Fig. 6a), whereas the maximum is around 9.5 ppmv in the reference run (Fig. $6 \mathbf{b}$ ). Thus, the difference between the maxima of the two runs is of the order of $5 \%$. Furthermore, there are not only decreased stratospheric ozone values for the coupled simulation, but additionally the maximum is shifted in its altitude. While for the reference run it is around $8 \mathrm{hPa}$, in the coupled run it is at about $10 \mathrm{hPa}$, i.e. at lower pressure levels, which also fits better to observations. On global average both effects (maximum decrease and maximum shift for the coupled run) lead to a slight increase in the vertically integrated ozone column (not shown here) in the coupled run. This increase is about 10 Dobson Units (DU), compared to a total amount around $300 \mathrm{DU}$, of which $90 \%$ origins from the stratosphere. However, it is not significant, especially as there could be a substantial influence also from the tropospheric chemistry, which was not interactive in our simulations. But in any case it shows, that there is increased mesospheric and tropospheric ozone in the coupled run, connected with weaker vertical gradients of the ozone concentrations in this simulation. This causes weaker vertical temperature gradients, ongoing with changes of superimposed thermal winds, and thus effects the extratropical troposphere-stratosphere vertical planetary wave propagation (following the theory by Charney and Drazin [35]) due to a changed vertical wind profile. In such a way also the troposphere-stratosphere coupling, which has the potential of changing the polar vortex regime (Perlwitz and Graf [36]), is modified for the coupled simulation compared to the reference simulation. 


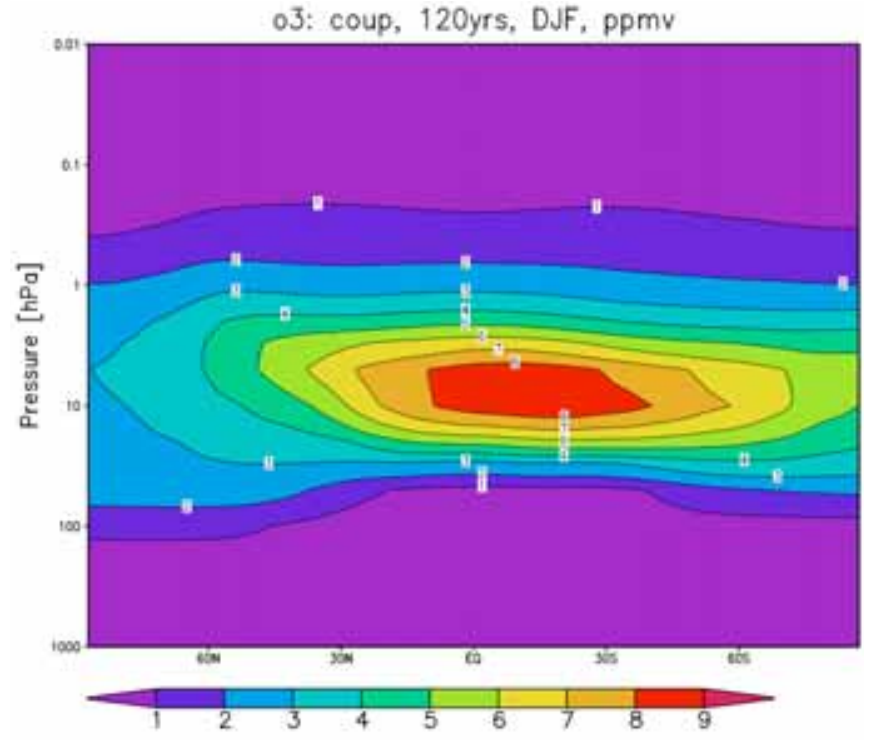

Fig. (6a). Zonal mean of the ozone concentration in [ppmv], averaged for DJF of the model years 31 to 150 . Coupled run.

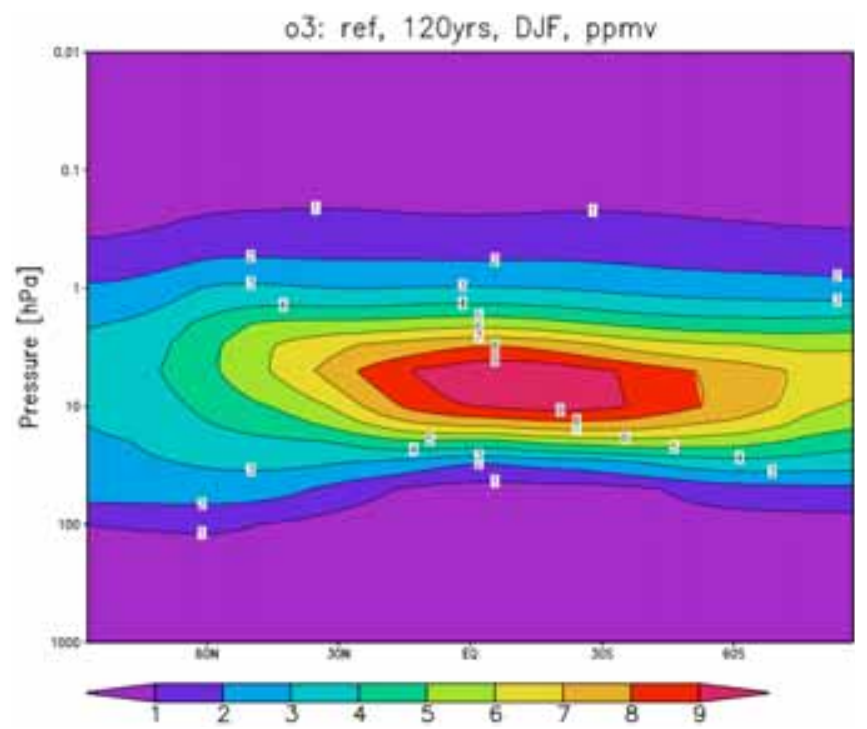

Fig. (6b). Zonal mean of the ozone concentration in [ppmv], averaged for DJF of the model years 31 to 150. Reference run.

The 500 and $200 \mathrm{hPa}$ plots of the geopotential height for the coupled run, the reference run and their differences (Fig. 7a-c and 8a-c) indicate a tropospheric warming in the tropics between the simulations (especially the differences (Figs. $\mathbf{7 c , 8 c )})$. The coupled run appears warmer than the reference run, which becomes obvious by differences in the geopotential height of around 20 to $25 \mathrm{gpm}$ at $500 \mathrm{hPa}$ and 60 to 70 $\mathrm{gpm}$ at $200 \mathrm{hPa}$. Furthermore, there are also indications for an associated warming in the polar winter troposphere over Northern America, again with the coupled run being warmer than the reference run. In contrast to that, a cooling facilitates in the midlatitudes over the Northern Atlantic and Northern Pacific oceans as a result of the chemistrydynamics coupling. The transition zone between this midlatitudinal cooling and the tropical warming appears with a wave like pattern, showing a wavenumber 4 behaviour. This might be a hint on a connected change of baroclinic instability mechanisms.

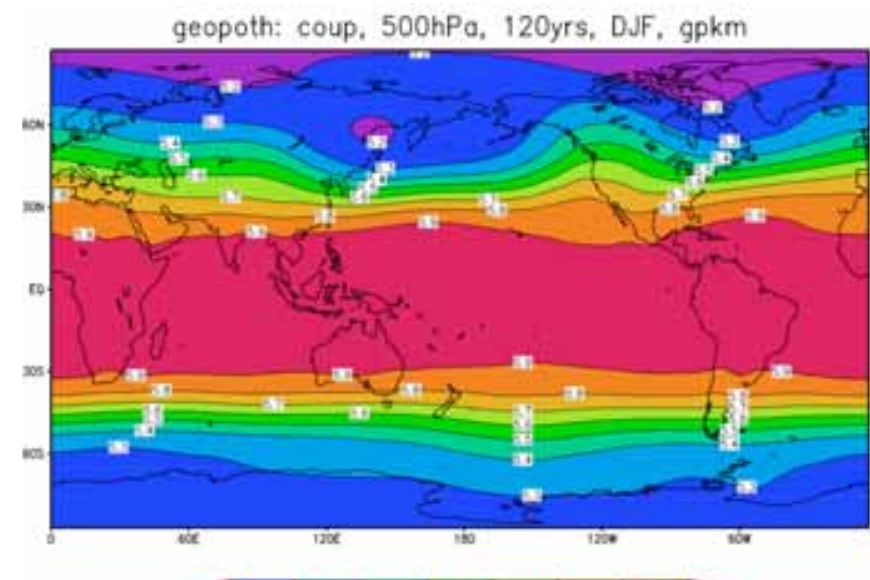

\begin{tabular}{llllllll}
\hline 1 & & & 1 & 1 & 1 & 1 & 1 \\
5.2 & 5.3 & 5.4 & 5.5 & 5.6 & 5.7 & 5.8 & 5.9
\end{tabular}

Fig. (7a). $500 \mathrm{hPa}$ geopotential height field in [gpkm], averaged for DJF of the model years 31 to 150 . Coupled run.

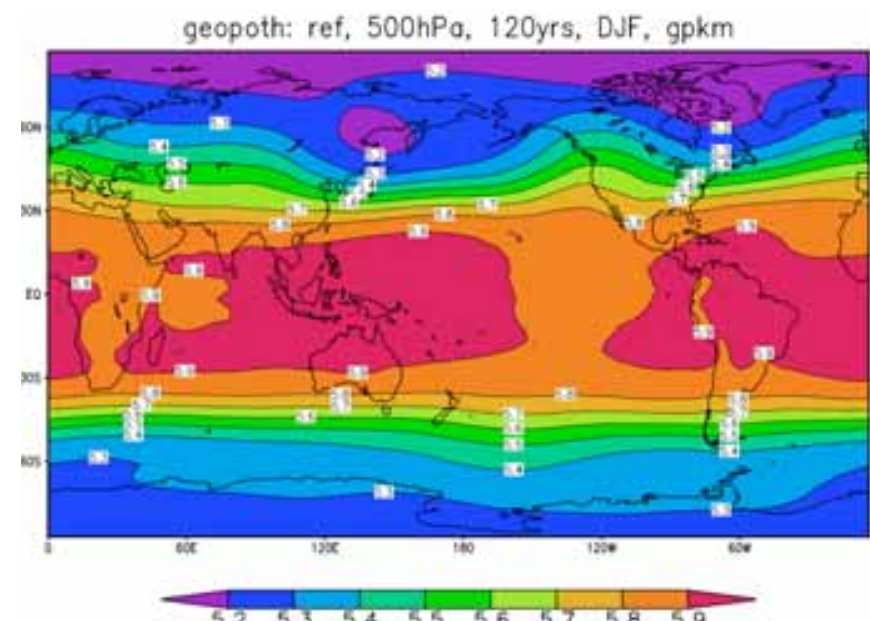

$\begin{array}{llllllll}5.2 & 5.3 & 5.4 & 5.5 & 5.6 & 5.7 & 5.8 & 5.9\end{array}$

Fig. (7b). $500 \mathrm{hPa}$ geopotential height field in [gpkm], averaged for DJF of the model years 31 to 150 . Reference run.

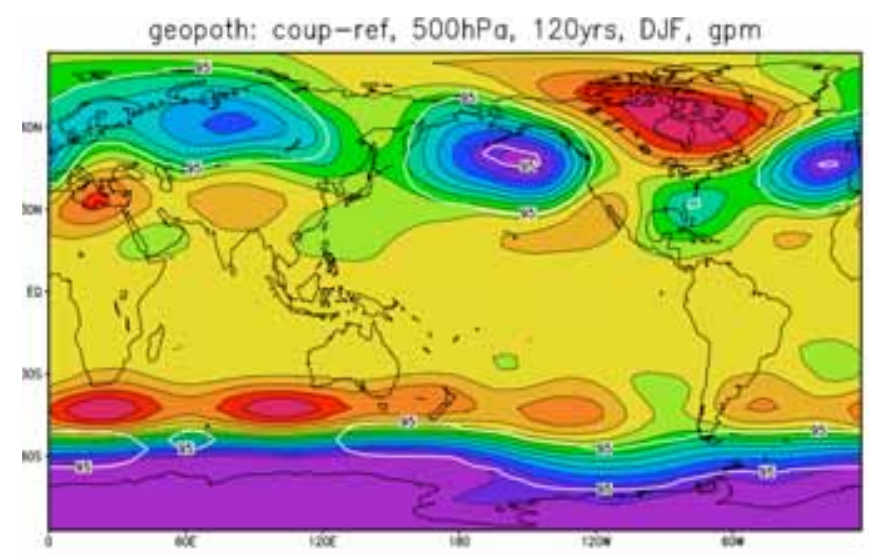

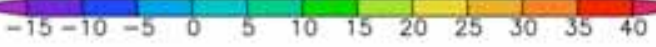

Fig. (7c). $500 \mathrm{hPa}$ geopotential height field in [gpm], averaged for DJF of the model years 31 to 150 . Differences between coupled-and reference run.

\subsection{Internal Variability within the Simulations}

Aiming to study the internal variability in the coupled and in the reference simulation, a principal component (PC) 


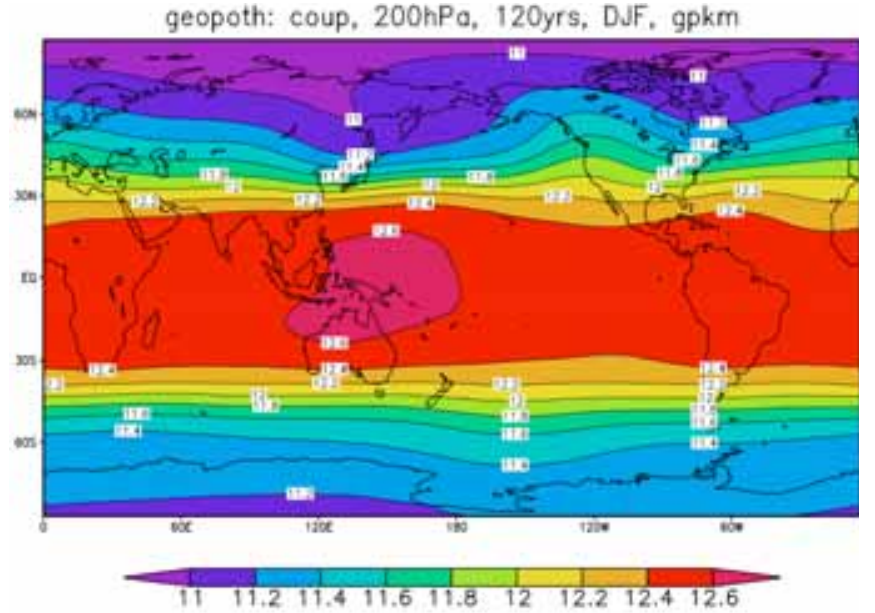

Fig. (8a). $200 \mathrm{hPa}$ geopotential height field in [gpkm], averaged for DJF of the model years 31 to 150 . Coupled run.

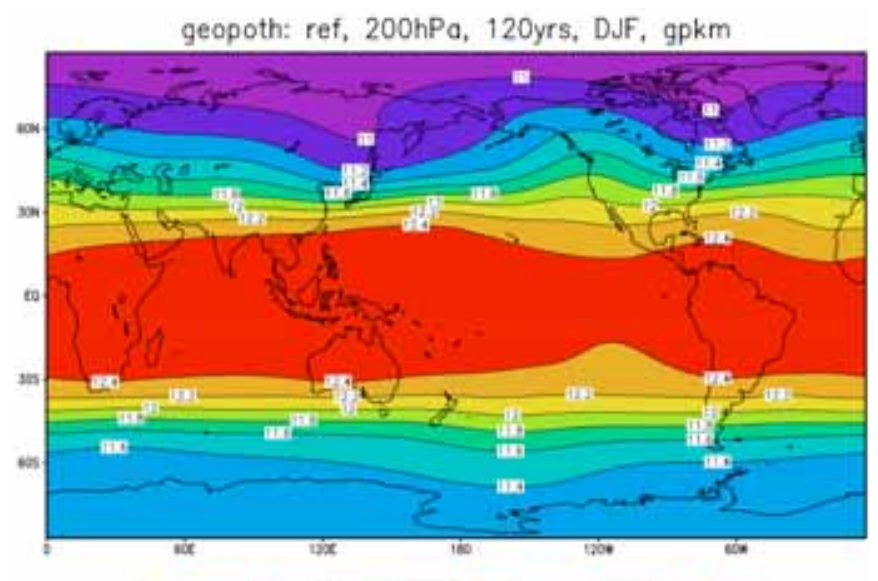

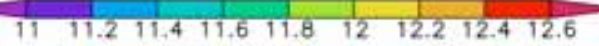

Fig. (8b). $200 \mathrm{hPa}$ geopotential height field in [gpkm], averaged for DJF of the model years 31 to 150 . Reference run.

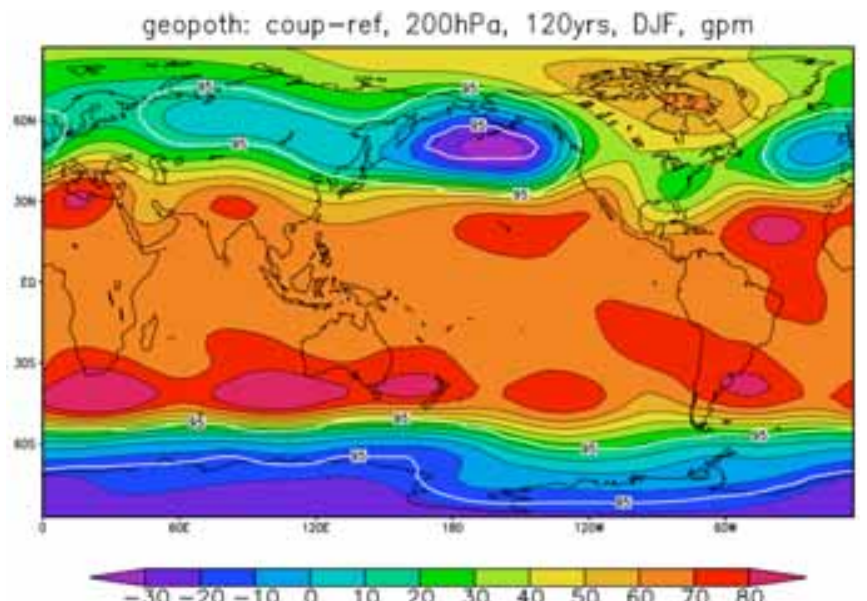

Fig. (8c). $200 \mathrm{hPa}$ geopotential height field in [g pm], averaged for DJF of the model years 31 to 150 . Differences between coupled- and reference run.

analysis was carried out (see von Storch and Zwiers [37] and references therein). The analysis was done for the same 120 years of each run, as used before (section 2.1). The first empirical orthogonal function (EOF1) and the associated PC (PC1, timeseries of weights for EOF1) on the $500 \mathrm{hPa}$ level

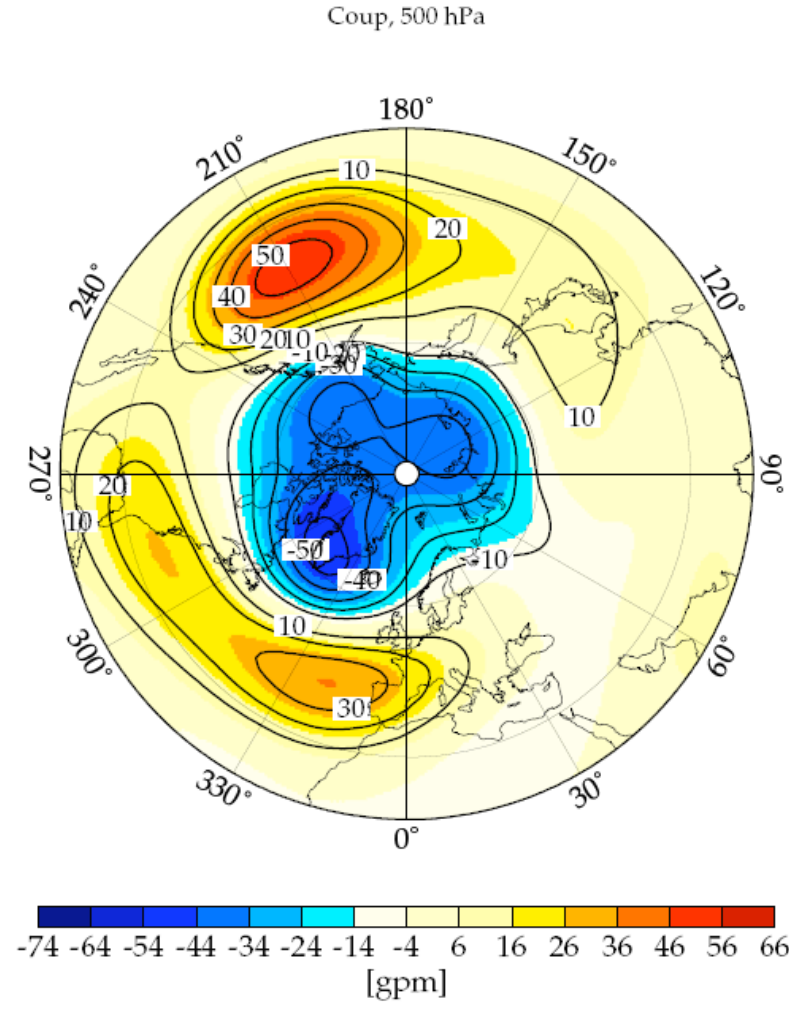

Fig. (9a). Principal Component Analysis (PCA) for DJF; model years 31 to 150 . Shown is the first Empirical Orthogonal Function (EOF1) of the $500 \mathrm{hPa}$ geopotential height field in [gpm]. Coupled run.

\section{Ref, $500 \mathrm{hPa}$}
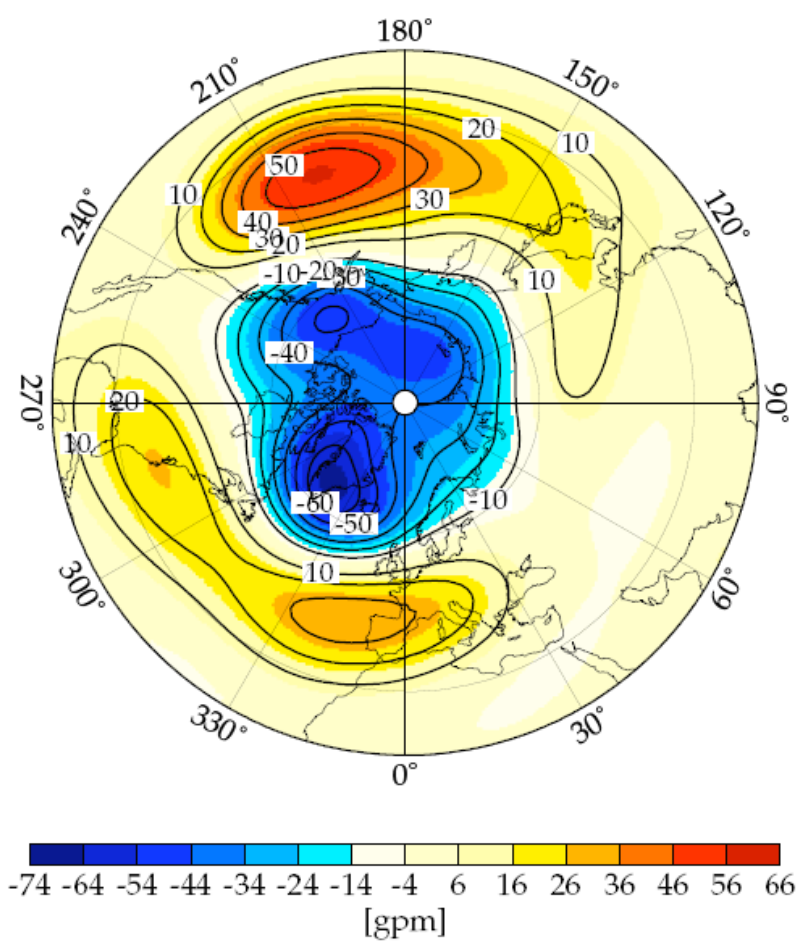

Fig. (9b). Principal Component Analysis (PCA) for DJF; model years 31 to 150. Shown is the first Empirical Orthogonal Function (EOF1) of the $500 \mathrm{hPa}$ geopotential height field in [gpm]. Reference run. 
are shown for both simulations (Figs. 9a,b and 10a,b), accomplished by the wavelet spectra of the timeseries (Fig. 11a,b). As for the mean mslp discussed above, the patterns themselves are in a good agreement. However, they explain different overall variances, with about $17 \%$ for the coupled run and $20 \%$ for the reference run, which generally hints on a weaker AO in the coupled run. The PC's (Fig. 10a,b) indicate clear interannual to decadal variability for both simulations. This can be seen even better in the wavelet spectra, where generally both runs show similar variability of the associated leading EOF's on all detected timescales.

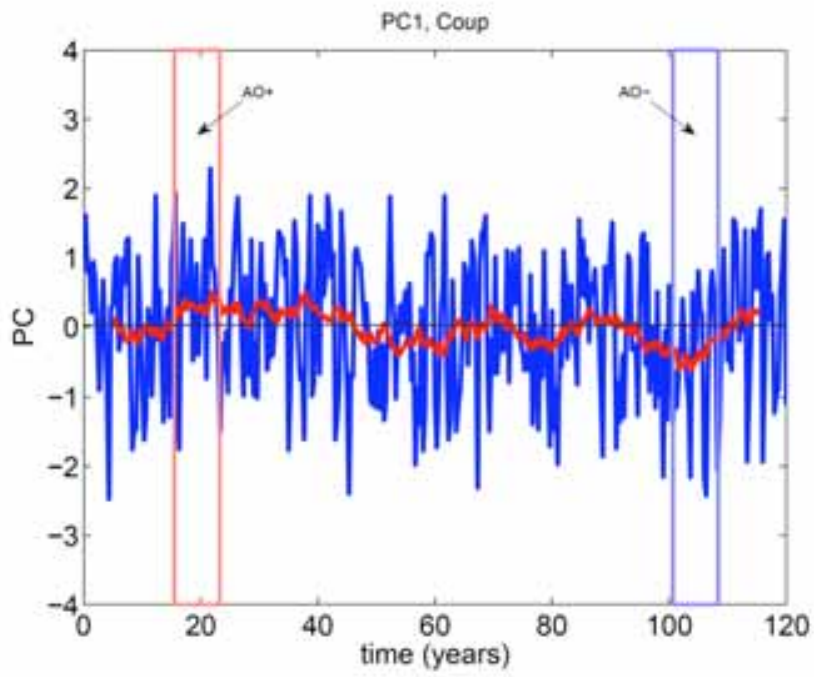

Fig. (10a). First Principal Component (PC1) of the $500 \mathrm{hPa}$ geopotential height field. DJF; model years 31 to 150 . PC1 is associated to EOF1 from Fig. (9a), giving the time evolution of this pattern. Additionally shown are the selected 8-year periods representing the positive and negative phase of the Arctic Oscillation (AO), which were used to create the Figs. (12-16). Coupled run.

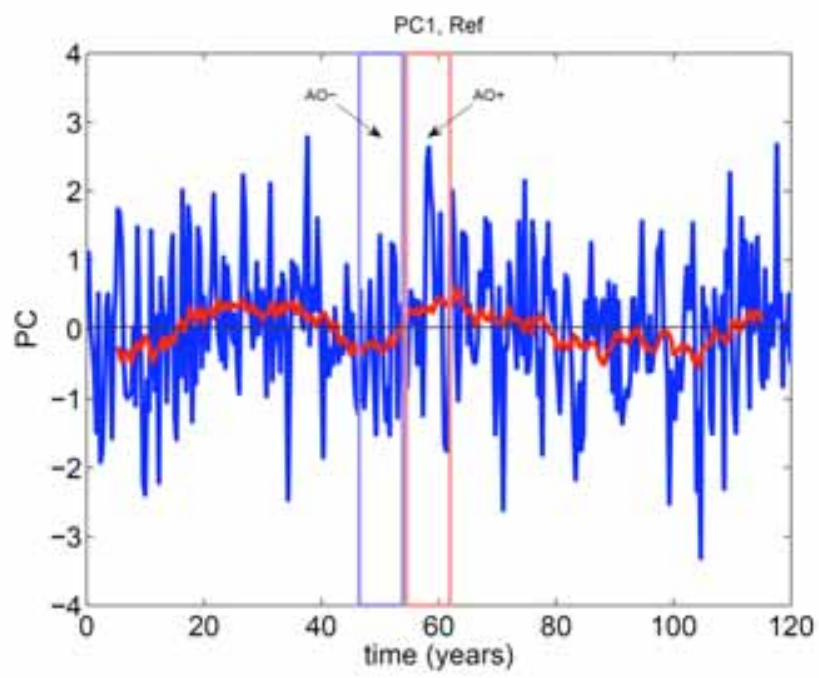

Fig. (10b). First Principal Component (PC1) of the $500 \mathrm{hPa}$ geopotential height field. DJF; model years 31 to 150 . PC1 is associated to EOF1 from Fig. (9b), giving the time evolution of this pattern. Additionally shown are the selected 8-year periods representing the positive and negative phase of the Arctic Oscillation (AO), which were used to create the Figs. (12-16). Reference run.

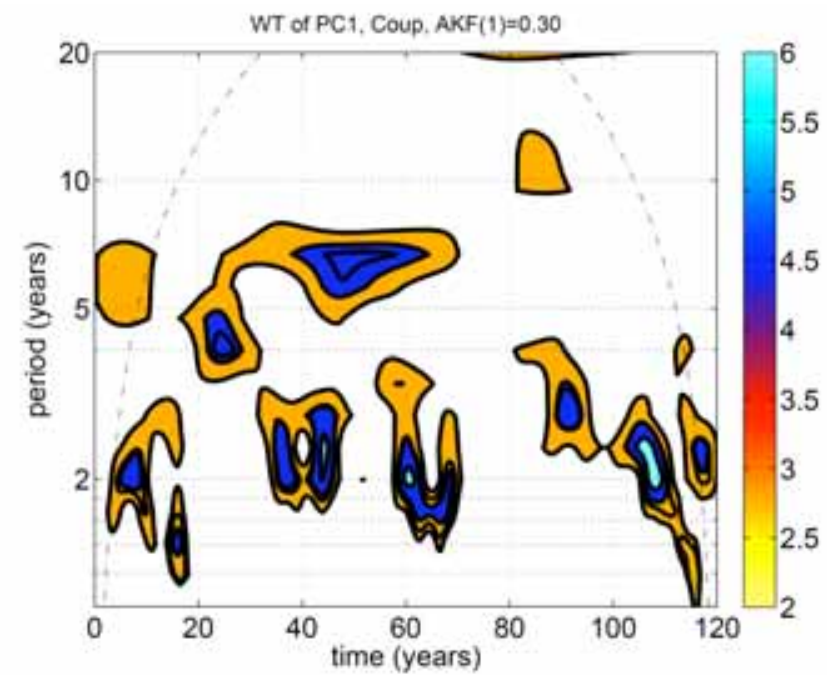

Fig. (11a). Wavelet power spectra of the 500 hpa geopotential height PC1 from Fig. (10a). Details in Fig. (3a,b). Coupled run.

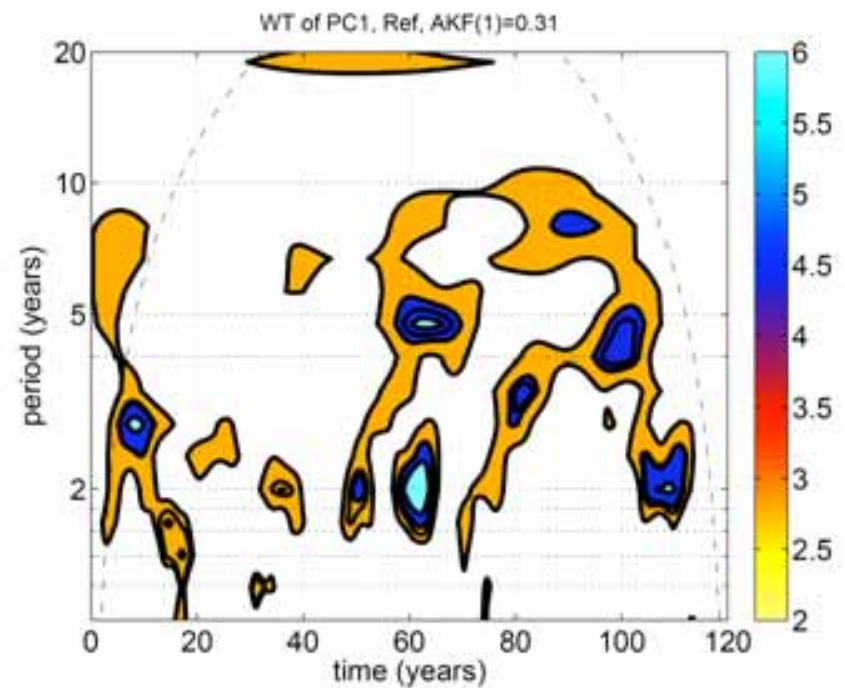

Fig. (11b). Wavelet power spectra of the 500 hpa geopotential height PC1 from Fig. (10b). Details in Fig. (3a,b). Reference run.

In terms of determining the internal variability in the model runs, comparing the differences between the PC phases for both simulations has the advantage to remove any direct setoffs, which were discussed in the previous section. Thus, coupling effects can be identified more clear. Additionally, since EOF1, which is used for splitting here, shows a clear AO-like occurance (Thompson and Wallace [32]), the $\mathrm{PC}+/ \mathrm{PC}$ - phases can directly be interpreted as the associated $\mathrm{AO}+/ \mathrm{AO}-$ phases, in order to provide a more physical discussion (Christiansen [38]). Thereby the AO+ phase is characterized as relatively cold in the stratosphere, with a strong polar stratospheric vortex as well as strong tropospheric midlatitudinal gradients, whereas the AO- phase is relatively warm in the stratosphere, with a weak polar stratospheric vortex and weaker tropospheric gradients in the midlatitudes. To illustrate both phases of each run, it was decided to pick the four 8-year periods shown in Fig. (10), using the $500 \mathrm{hPa}$ PC's:

- $\quad$ Years 16 to 23 of the coupled run, representing the positive phase of the $\mathrm{PC} / \mathrm{AO}$.

- $\quad$ Years 101 to 108 of the coupled run, representing the negative phase of the PC/AO. 
- $\quad$ Years 47 to 54 of the reference run, representing the negative phase of the PC/AO.

- Years 55 to 62 of the reference run, representing the positive phase of the PC/AO.

Starting with the zonal means of the zonal windspeed, it can be stated, that a similar pattern compared to the run-torun differences (section 3.1, Fig. 5c) holds for the tropospheric differences between the AO phases in both simulations (Fig. 12a,b), but with the opposite sign. The weakening of the subtropical tropospheric jets in the $\mathrm{AO}+$ phase occurs rather in the winter hemisphere $(\mathrm{NH})$ and for the coupled run. In the polar stratosphere and mesosphere the jet becomes stronger in the $\mathrm{AO}+$ phase, which is consistent for both runs, but with 2 to $4 \mathrm{~m} / \mathrm{s}$ much weaker in case of the coupled run (Fig. 12a), compared to the reference run with differences up to $16 \mathrm{~m} / \mathrm{s}$ (Fig. 12b).

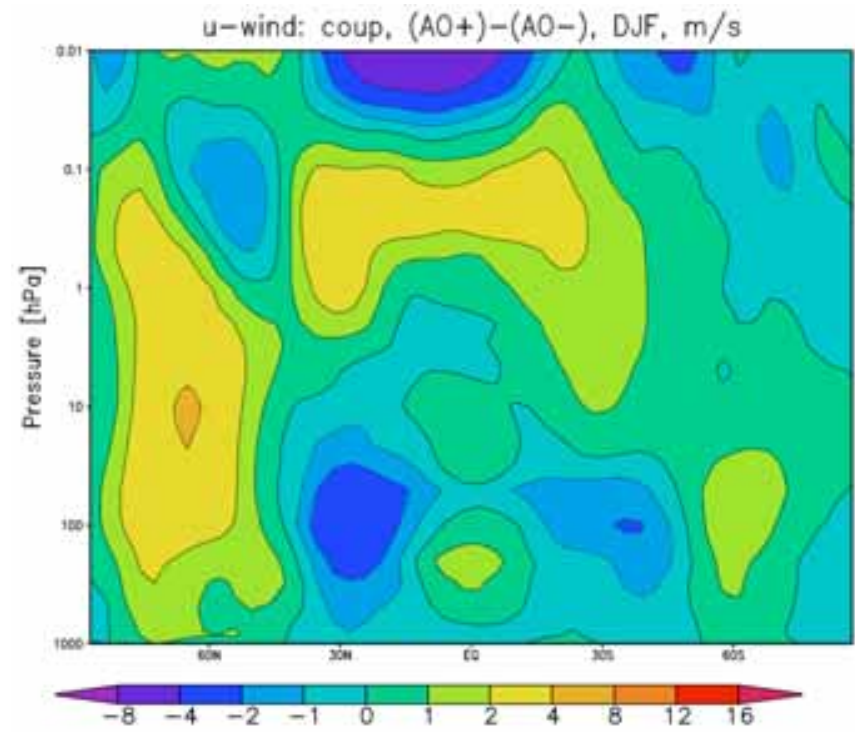

Fig. (12a). Zonal mean of the zonal wind $u$ in $[\mathrm{m} / \mathrm{s}]$, averaged for DJF of always 8 model years representing the positive and negative phase of the Arctic Oscillation (AO). For the exact years see Fig. (10a). Differences 'AO+ minus AO-' for the coupled run.

In the Fig. (13a,b), which present the internal ozone differences between the AO phases for both runs, a shift of up to $-400 \mathrm{ppbv}$ for the reference run and $-300 \mathrm{ppbv}$ for the coupled run is obvious in the polar stratosphere at $\mathrm{AO}+$ conditions. This corresponds to about $10 \%$ of the mean values in this region, and can directly be understood, since under strong stratospheric vortex conditions, connected to the AO+ phase in the troposphere, less stratospheric ozone can be transported towards the winter pole due to weaker planetary waves. A second feature can be seen in the tropics at the ozone maximum altitudes, where a slight weakening $(\sim 100$ ppbv) of the mean values can be detected for the AO+ phase. This weakening is also consistent for both simulations, while the differences between the runs, namely in the summer hemisphere, are not significant.

A tropical tropospheric signal occurs between the AO phases for the coupled run, determining a cooling at $\mathrm{AO}+$ conditions. This is underlined by the 500 and $200 \mathrm{hPa}$ plots of the geopotential height (Figs. 14a and 15a), which show

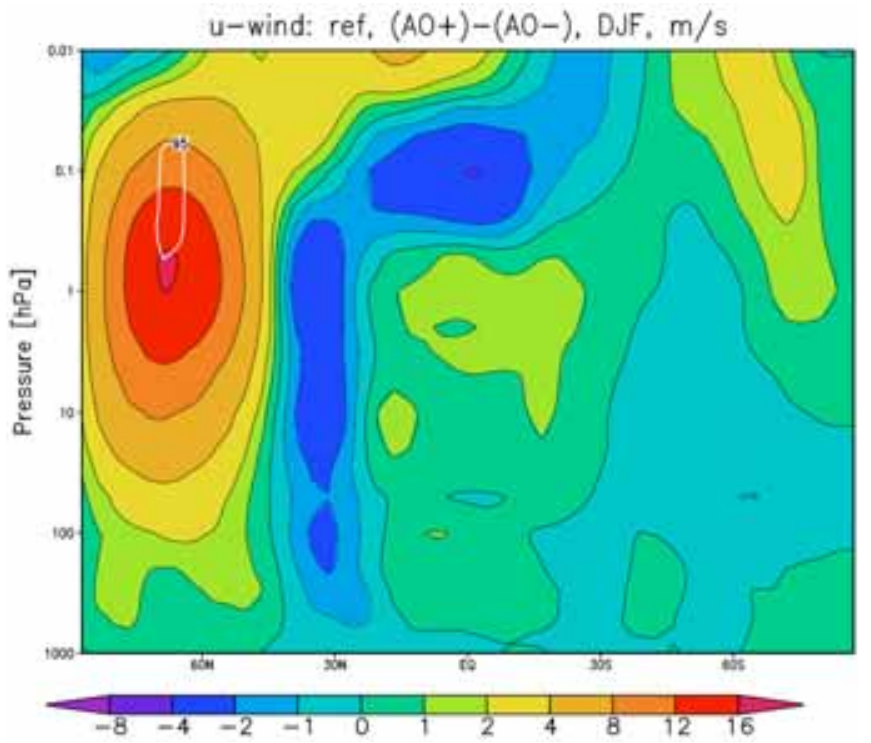

Fig. (12b). Zonal mean of the zonal wind $\mathrm{u}$ in $[\mathrm{m} / \mathrm{s}]$, averaged for DJF of always 8 model years representing the positive and negative phase of the Arctic Oscillation (AO). For the exact years see Fig. (10b). Differences 'AO+ minus AO-' for the reference run.

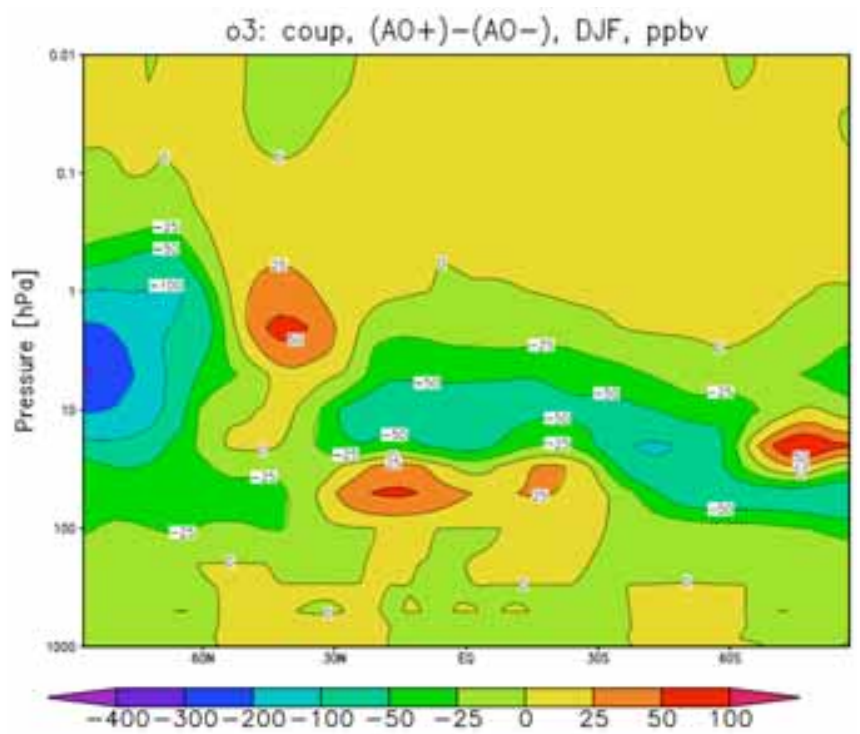

Fig. (13a). Zonal mean of the ozone concentration in [ppbv]. Averages as in Fig. (12a,b). Differences 'AO+ minus AO-' for the coupled run.

significant differences of around $20 \mathrm{gpm}$ at $500 \mathrm{hPa}$ and 40 gpm at $200 \mathrm{hPa}$. For the reference run (Figs. 14b and 15b) the tropical setoff between the two AO phases of about 10 gpm is not significant, but as for the coupled run an associated effect in the polar winter $(\mathrm{NH})$ atmosphere is indicated, which is strongest over the north-western parts of Northern America and northward to the pole. In the coupled run this effect distributes more zonal symmetric (Figs. 14a and 15a), although again with emphasis on Northern America. In both simulations the corresponding significance exceeds 95\%, while the particular midlatitudinal differences, favouring a warming in the $\mathrm{AO}+$ phase, are rather noisy.

Nevertheless it should be noted here, that the concrete locations of the geopotential height maxima for the coupled 


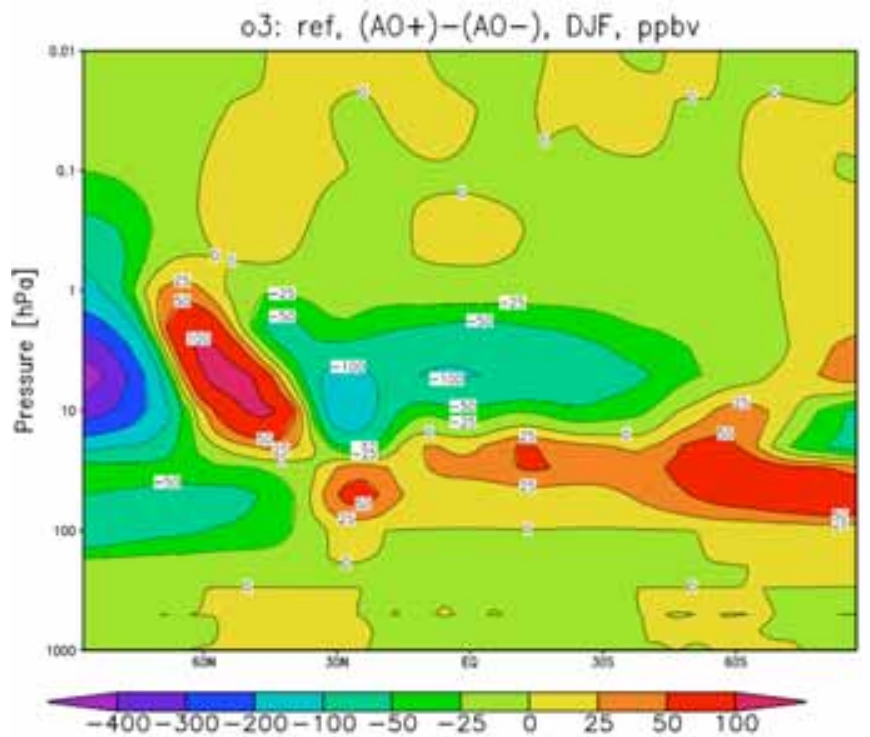

Fig. (13b). Zonal mean of the ozone concentration in [ppbv]. Averages as in Fig. (12a,b). Differences 'AO+ minus AO-' for the reference run.

and the reference run (Figs. 14a,b and 15a,b), although not forced in any manner, occur in remarkable agreement for the comparison of both simulations. On the other hand the strength of the particular maxima differs clearly, which suggests strong regional feedbacks in the coupled run due to the interactive ozone coupling. Additionally, the overall appearance of the difference patterns with cooling, warming and cooling for the $\mathrm{AO}+$ phase can be taken as an indication for a connected enhancement of baroclinic waves in this phase of both runs. Such a behaviour would agree with physical expectations, since the strenght of the tropospheric zonal mean flow is directly linked to the polar stratospheric vortex, which is also stronger and less disturbed in this AO phase. Furthermore, the differences in the Figs. (14a,b and 15a,b) again suggest a wavenumber 4 behaviour, which is supported also by the geopotential height meridional means: The $\mathrm{AO}+/ \mathrm{AO}-$ differences likewise differ between the simulations showing a wavenumber 4 in the troposphere and a wavenumber 2 in the stratosphere and mesosphere (Fig. 16). At the same time this provides an instructive example on troposphere-stratosphere coupling, since obviously (mid- and highlatitudinal) changes of either tropospheric circulation, characterized by planetary and baroclinic waves, or stratospheric/mesospheric circulation, where mainly planetary waves dominate, are directly linked.

\section{DISCUSSION AND CONCLUSIONS}

For this study we applied the fully coupled AOGCM ECHO-GiSP with interactive stratospheric chemistry to perform 2 long term simulations of 150 years each. One of the runs ('reference run') treated the chemistry scheme as a passive part of the model, i.e. itself depending on the dynamical model variables, but without any feedback to them. In contrary, for the second run ('coupled run') the simulated ozone concentrations were considered interactively within the radiation scheme instead of the prescribed parameterizations used in the reference run. Thereby in the coupled run the model was not (arbitrarily) forced away from its internal

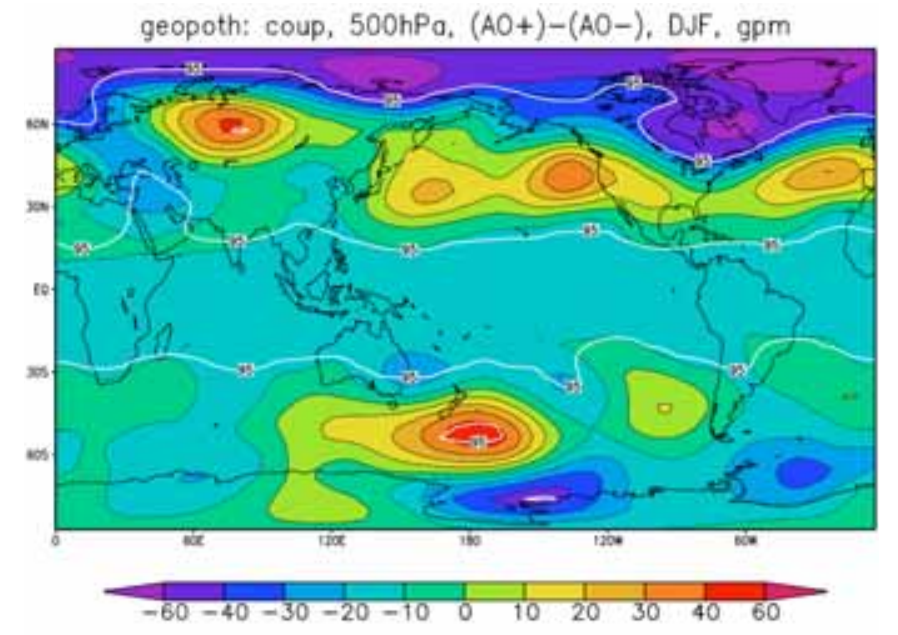

Fig. (14a). $500 \mathrm{hPa}$ geopotential height field in [gpm]. Averages as in Fig. (12a,b). Differences 'AO+ minus AO-' for the coupled run.

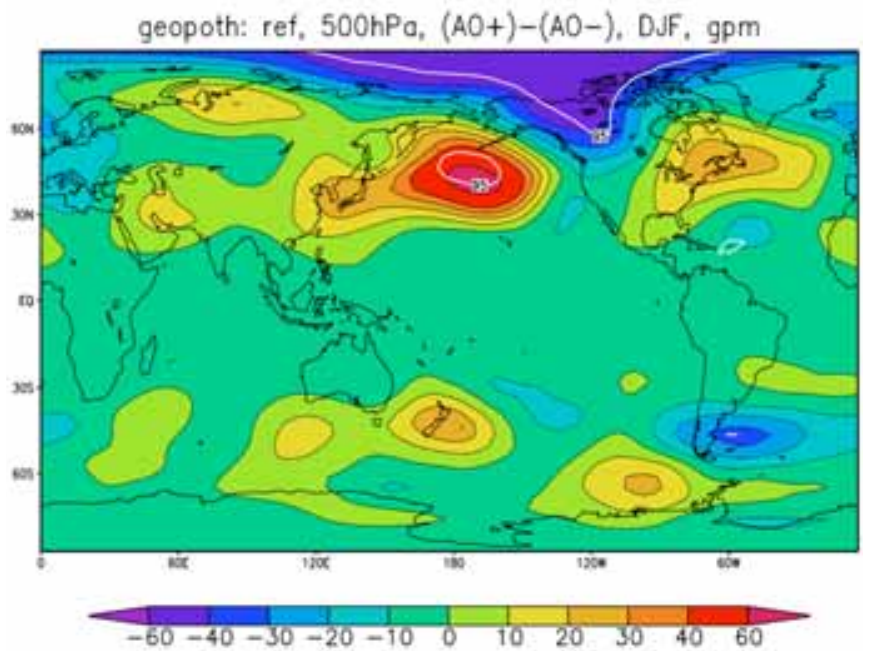

Fig. (14b). $500 \mathrm{hPa}$ geopotential height field in [gpm]. Averages as in Fig. (12a,b). Differences 'AO+ minus AO-' for the reference run.

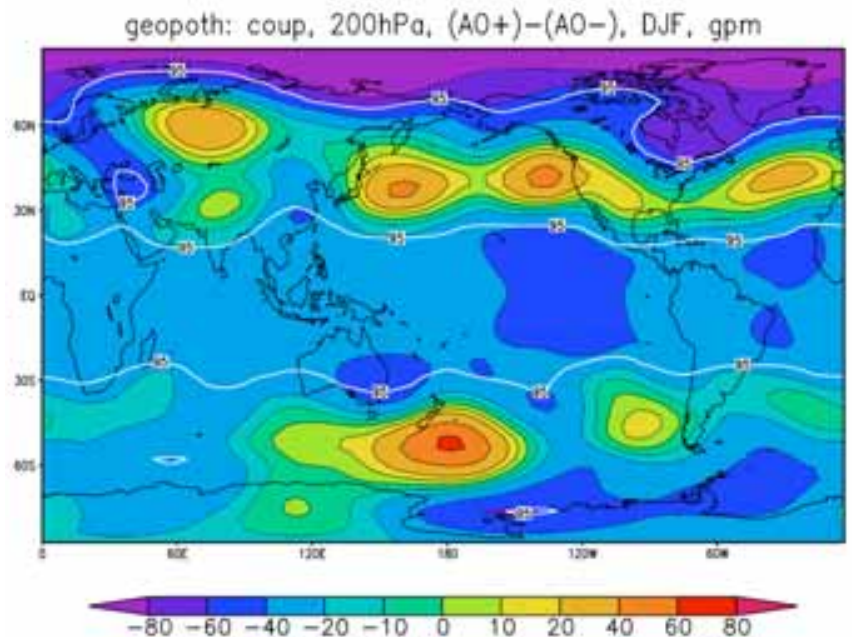

Fig. (15a). $200 \mathrm{hPa}$ geopotential height field in [gpm]. Averages as in Fig. (12a,b). Differences 'AO+ minus AO-' for the coupled run. 


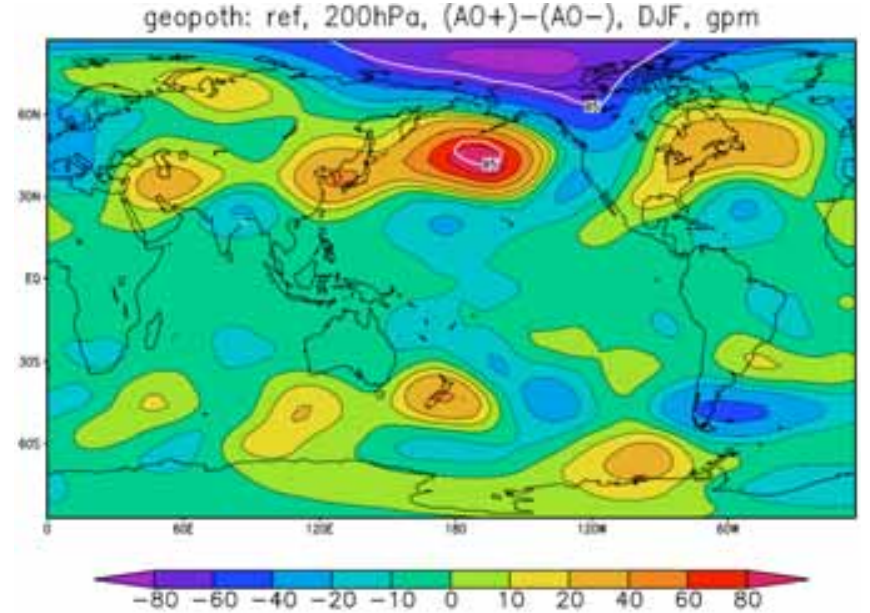

Fig. (15b). $200 \mathrm{hPa}$ geopotential height field in [gpm]. Averages as in Fig. (12a,b). Differences 'AO+ minus AO-' for the reference run.

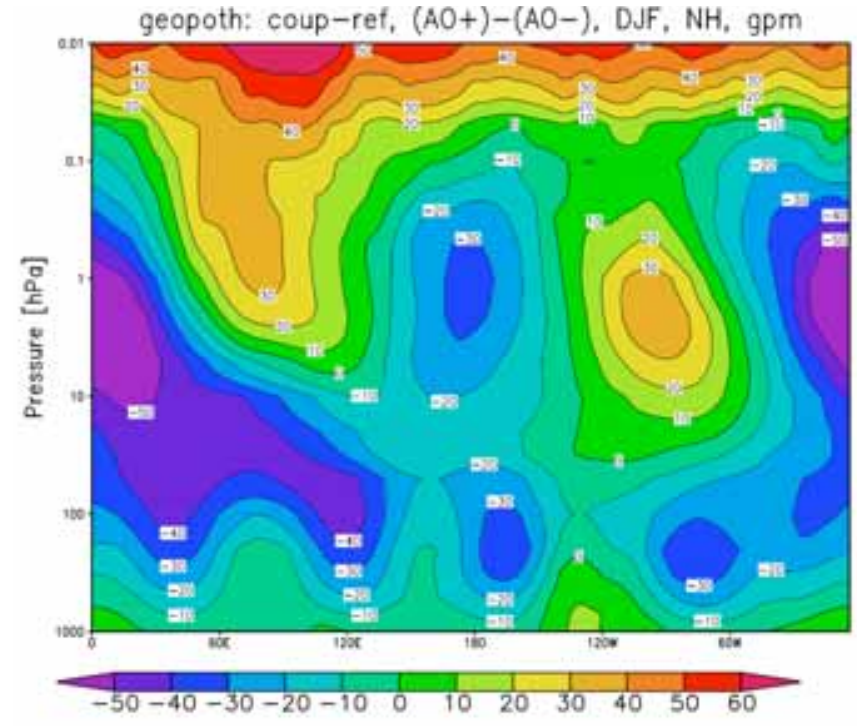

Fig. (16). Meridional mean of the northern hemisphere geopotential heights in [gpm]. Averages as in Fig. (12a,b). Differences between coupled- and reference run of the differences ' $\mathrm{AO}+$ minus AO-' within the runs.

physical-chemical equilibrium state any more, which is essential for understanding the dynamical changes between the simulations.

First, following a spin-up period of 30 model years, climatologies of 120 model years were examined for each run. As a main result we showed, that in comparison with the reference run the coupled run tends to the negative phase of the Arctic Oscillation (AO) mode. Consistent with this we detected two effects, leading to a weakened tropospheric zonal mean flow and an enhanced tropospheric meridional mean circulation for the coupled simulation. One effect is a tropospheric warming over Northern America and towards the pole in the coupled simulation, while at the same time a midlatitudinal cooling occurs, especially over the northern Pacific and northern Atlantic. This weakens the temperature gradient between high- and midlatitudes, and thus also the tropospheric zonal mean flow. The second effect is a tropospheric tropical warming, causing enhanced tropical ascent and thereby speeding up the tropospheric meridional mean circulation in the coupled run.

Furthermore, we also determined a strenghtening of the subtropical tropospheric jet for the coupled run, which we attribute to the strengthening of the tropospheric meridional mean circulation. At the same time a weakening of the polar stratospheric jet occurs, most probably due to an increase of the tropospheric planetary wave activity. We did not show this here, but present results of an appropriate Fourier analysis within Brand et al. [39]. Nevertheless, it is a widely recognized fact, that a weakening of the tropospheric zonal mean flow as in our coupled run is related to enhanced wave activity in general (This includes not only planetary- but also synoptic scale waves.). On the other hand the maximum available wave energy is also depending on the weakening midlatitudinal meridional temperature gradient. Thus, the troposphere-stratosphere coupling, which is enhanced in the coupled run due to the wave forcing by vertical propagating planetary waves from the troposphere into the stratosphere (Charney and Drazin [35]), can reach an equilibrium even if there is a positive feedback between the weakening of the polar stratospheric vortex and the wave forcing by ascending tropospheric planetary waves, as one could doubt following Baldwin and Dunkerton [40], Christiansen [41] or Wittmann et al. [42]: All of these authors suggested a stratospheric control of the tropospheric circulation. Our results underline, that this control mechanism, if existing, is also clearly effected by stratospheric ozone feedbacks.

As a second step we discussed the internal variability of both runs between the AO phases based on an EOF analysis of the $500 \mathrm{hPa}$ geopotential height. Generally, our results show a stronger polar vortex in the stratosphere and mesosphere for the $\mathrm{AO}+$ phase, but in case of the coupled run this effect is much weaker compared to the reference run. This suggests an enhanced tropospheric variability within the coupled simulation, while at the same time the stratospheric/mesospheric variability appears to be declined. However, similar to the circulation changes between the coupledand the reference run it is convenient also to attribute the weakening of the polar stratospheric vortex in the AO-phase as a result of enhanced tropospheric wave activity. This leads to more upward propagating tropospheric planetary waves, which slow down the stratospheric zonal mean flow and thus, in turn, speed up the stratospheric parts of the stratospheric-mesospheric Brewer-Dobson meridional mean circulation (Eichelberger and Hartmann [43]). Our results illustrate this by showing an enhanced polar and reduced tropical stratospheric ozone accumulation in the AO-phase, although the characteristic of this changes differs between the simulations. In agreement with the above conclusions the effect is stronger for the reference run, indicating more variability of the stratospheric-mesospheric Brewer-Dobson meridional mean circulation there compared to the coupled run.

The geopotential height differences between $\mathrm{AO}+$ and AO- for the coupled- and for the reference simulation also show results similar to the differences between the runs. A cooling over high latitudes, especially over the north-western North America, and a warming in midlatitudes for the $\mathrm{AO}+$ phase appears in both runs, where the effect is slightly stronger for the coupled run. This might be connected with the different AO mean states of the two runs, namely the 
tendency of the coupled run towards the negative phase of the AO. Additionally, only the coupled run shows a significant cooling in the tropical troposphere for $\mathrm{AO}+$, which therefore seems to be linked directly with the stratospheric interactive chemistry. In particular we show and discuss this in Brand et al. [39].

Summarizing, both simulations carried out for this study show significant differences with respect to dynamic features as the appearance and variability of the tropospheric and stratospheric jets or the connected wave activity. In the troposphere, the coupled run, including interactive chemistry feedbacks, tends to the negative phase of the AO, including stronger subtropical jets and a stronger meridional mean circulation. This is connected with warmer stratospheric conditions and a weaker, more disturbed polar stratospheric vortex. The coupled run generally occurs with enhanced tropospheric variability, whereas the stratospheric and mesospheric variability shows the opposite behaviour. On the other hand, the overall interannual and decadal variability of both runs appear similar, although in particular may be with slightly more activity on interannual timescales within the coupled simulation. However, the so far results of this study are promising towards a better understanding of general coupling effects in comprehensive atmosphere(-chemistry)ocean-sea ice models.

\section{ACKNOWLEDGEMENTS}

We would like to thank U. Cubasch and I. Kirchner from FU Berlin (ECHO-G), MPI for Chemistry Mainz (MECCA), and R. Ruhnke and M. Wiehle from FZ Karlsruhe (chemistry boundary conditions) for providing model components or necessary data. We are also grateful to K. Ketelsen for his support in programming key routines of the model environment, and to S. Erxleben for technical support and preparing the graphics. K.D. and S.B. participated in the Virtual Institute 'Pole-Equator-Pole' kept by the German Helmholtz Association.

\section{REFERENCES}

[1] Manzini E, Bengtsson L. Stratospheric climate and variability from a general circulation model and observations. Clim Dyn 1996; 12: 615-639.

[2] Shindell DT, Rind D, Lonergan P. Climate Change and the Middle Atmosphere. Part IV: Ozone Response to Doubled $\mathrm{CO}_{2}$. J Clim 1998a; 11: 895-918.

[3] Shindell DT, Rind D, Lonergan P. Increased polar stratospheric ozone losses and delayed eventual recovery owing to increasing greenhouse gas concentrations. Nature 1998b; 392: 589-592.

[4] Eckman RS, Grose WL, Turner RE, Blackshear WT. Polar ozone depletion: A three-dimensional chemical modeling study of its long-term global impact. J Geophys Res 1996; 101: 22977-22990.

[5] Austin J, Knight J, Butchart N. Three-dimensional chemical model simulations of the ozone layer: 1979-2015. Q J R Meteorol Soc 2000; 126: 1533-1556.

[6] Austin J. A Three-Dimensional Coupled Chemistry-Climate Model Simulation of Past Stratospheric Trends. J Atmos Sci 2002; 59: 218-232.

[7] Dameris M, Grewe V, Hein R, Schnadt C, Bruehl C, Steil B. Assessment of the future development of the ozone layer. Geophys Res Lett 1998; 25: 3579-3582.

[8] Hein R, Dameris M, Schnadt C, et al. Results of an interactively coupled atmospheric chemistry general circulation model: Comparison with observations. Ann Geophys 2001; 19: 435-457.

[9] Holton JR, Haynes PH, McIntyre ME, Douglass AR, Rood RB, Pfister L. Stratosphere-troposphere exchange. Rev Geophys 1995; 33: 403-440.
[10] Haynes PH, McIntyre ME, Shepherd TG, Marks CJ, Shine KP. On the 'Downward Control' of Extratropical Diabatic Circulations by Eddy-Induced Mean Zonal Forces. J Atmos Sci 1991; 48: 651-680.

[11] Rind D, Lerner J, Shah K, Suozzo R. Use of on-line tracers as a diagnostic tool in general circulation model development 2. Transport between the troposphere and stratosphere. J Geophys Res 1999; 104: 9151-9168.

[12] Steil B, Bruehl C, Manzini E, et al. A new interactive chemistryclimate model: 1 . Present-day climatology and interannual variability of the middle atmosphere using the model and 9 years of HALOE/UARS data. J Geophys Res (Atm.) 2003; 108: 11.

[13] Latif M, Arpe K, Roeckner E. Oceanic control of decadal North Atlantic sea level pressure variability in winter. Geophys Res Lett 2000; 27: 727-730.

[14] von Storch JS. Signatures of Air-Sea Interactions in a Coupled Atmosphere-Ocean GCM. J Clim 2000; 13: 3361-3379.

[15] Dethloff K, Rinke A, Benkel A, et al. A dynamical link between the Arctic and the global climate system. Geophys Res Lett 2006; 33: 3703 .

[16] Rinke A, Maslowski W, Dethloff K, Clement J. Influence of sea ice on the atmosphere: A study with an Arctic atmospheric regional climate model. J Geophys Res (Atm) 2006; 111: 16103.

[17] Huebener H, Cubasch U, Langematz U, et al. Ensemble climate simulations using a fully coupled ocean-troposphere-stratosphere general circulation model. Phil Trans R Soc A 2007; 365: 20892101

[18] Legutke S, Voss R. The Hamburg Atmosphere-Ocean Coupled Circulation Model ECHO-G. Technical Report No. 18, 1999; ISSN 0940-9327, DKRZ Hamburg, Germany.

[19] Sander R, Kerkweg A, Joeckel P, Lelieveld J. Technical note: The new comprehensive atmospheric chemistry module MECCA. Atmos Chem Phys 2005; 5: 445-450.

[20] Valcke S, Terray L, A. Piacentini A. The OASIS coupler user guide version 2.4. Technical Report TR/CGMC/00-10, 2000; CERFACS, Toulouse, France.

[21] Roeckner E, Arpe K, Bengtsson L, et al. The atmospheric general circulation model ECHAM4: Model description and simulation of present-day climate. Report No. 218, 1996; MPI for Met. Hamburg, Germany.

[22] Manzini E, McFarlane NA, McLandress C. Impact of the Doppler spread parameterization on the simulation of the middle atmosphere circulation using the MA/ECHAM4 general circulation model. J Geophys Res 1997; 102: 25751-25762.

[23] Manzini E, McFarlane NA. The effect of varying the source spectrum of a gravity wave parameterization in a middle atmosphere general circulation model. J Geophys Res 1998; 103: 31523-31540.

[24] Wolff JO, Maier-Reimer E, Legutke S. The Hamburg Ocean Primitive Equation Model HOPE. Technical Report No. 13, 1997; ISSN 0940-9327, DKRZ Hamburg, Germany.

[25] Legutke S, Maier-Reimer E. Climatology of the HOPE-G Global Ocean - Sea Ice General Circulation Model. Technical Report No. 21, 1999; ISSN 0940-9327, DKRZ Hamburg, Germany.

[26] Damian-Iordache V, Sandu A. KPP - A symbolic preprocessor for chemistry kinetics - User's guide. Technical report, 1995; Dep Math Univ Iowa.

[27] Damian V, Sandu A, Damian M, Potra F, Carmichael GR. The kinetic preprocessor KPP: A software environment for solving chemical kinetics. Comput Chem Eng 2002; 26: 1567-1579.

[28] Landgraf J, Crutzen PJ. An Efficient Method for Online Calculations of Photolysis and Heating Rates. J Atmos Sci 1998; 55: 863878.

[29] Rasch PJ, Williamson DL. On shape-preserving interpolation and semi-Lagrangian transport. SIAM J Sci Stat Comput 1990; 11: 656687.

[30] Morcrette JJ. Radiation and cloud radiative properties in the European Center for Medium Range Weather Forecasts forecasting system. J Geophys Res 1991; 96: 9121-9132.

[31] Kouker W, Langbein I, Reddmann T, Ruhnke R. The Karlsruhe Simulation Model of the Middle Atmosphere (KASIMA), Version 2. Wiss. Berichte, FZKA 6278, 1999; Forschungszentrum Karlsruhe, Germany.

[32] Thompson DWJ, Wallace JM. The Arctic Oscillation signature in the wintertime geopotential height and temperature fields. Geophys Res Lett 1998; 25: 1297-1300.

[33] Torrence C, Compo GP. A practical guide to wavelet analysis. Bull Amer Meteor Soc 1998; 79: 61-78. 
[34] Hurrell JW. Decadal Trends in the North Atlantic Oscillation: Regional Temperatures and Precipitation. Science 1995; 269: 676679.

[35] Charney JG, Drazin PG. Propagation of planetary-scale disturbances from the lower into the upper atmosphere. J Geophys Res 1961; 66: 83-109.

[36] Perlwitz J, Graf HF. Troposphere-stratosphere dynamic coupling under strong and weak polar vortex conditions. Geophys Res Lett 2001; 28: 271-274.

[37] von Storch H, Zwiers FW. Statistical analysis in climate research. Cambridge University Press 1999, Cambridge.

[38] Christiansen B. On the physical nature of the Arctic Oscillation. Geophys Res Lett 2002; 29: 52-1.
[39] Brand S, Dethloff K, Handorf D. Tropospheric circulation sensitivity to an interactive stratospheric ozone. Geophys Res Lett 2007; submitted.

[40] Baldwin MP, Dunkerton TJ. Propagation of the Arctic Oscillation from the stratosphere to the troposphere. J Geophys Res 1999; 104: 30937-30946.

[41] Christiansen B. Downward Propagation of the Arctic Oscillation: Mechanism and Control. EGS XXVII General Assembly, Nice, 2126 April 2002, Abstract \# 4137; 27: 4137.

[42] Wittman MAH, Polvani LM, Scott RK, Charlton AJ. Stratospheric influence on baroclinic lifecycles and its connection to the Arctic Oscillation. Geophys Res Lett 2004; 31: 16113.

[43] Eichelberger SJ, Hartmann DL. Changes in the strength of the Brewer-Dobson circulation in a simple AGCM. Geophys Res Lett 2005; 32: 15807 . 